https://doi.org/10.15407/ujpe63.8.683

I.V. SERDEHA, V.I. GRYGORUK, G.S. FELINSKYI

Taras Shevchenko National University of Kyiv,

Faculty of Radio Physics, Electronics and Computer Systems

(4g, Prosp. Academician Glushkov, Kyiv 03143, Ukraine; e-mail: ivserdeha@gmail.com)

\title{
SPECTROSCOPIC FEATURES \\ OF RAMAN GAIN PROFILES IN SINGLE-MODE FIBERS BASED ON SILICA GLASS
}

\begin{abstract}
The spectroscopic analysis of the frequency distribution of the amplification of optical radiation due to the Raman effect (Raman gain profile) in single-mode fibers based on silica glass has been carried out in the region of Stokes frequency shifts from 0 to $1400 \mathrm{~cm}^{-1}$. The Raman gain profiles are determined from the experimental spectra of spontaneous scattering for widespread fibers, namely for pure $\mathrm{SiO}_{2}, \mathrm{GeO}_{2}, \mathrm{P}_{2} \mathrm{O}_{5}$, and $\mathrm{TiO}_{2}$ doped fibers. The analytic expressions of the Raman gain profiles are given. They are obtained, by using the Gaussian decomposition by means of 11-12 modes, and the experimental profile is approximated with an accuracy of not less than $0.3 \%$. The decomposition results are analyzed in terms of the fundamental oscillatory dynamics of molecular nanocomplexes in amorphous glass, as well as in the application aspect of the modeling of photonics devices. Examples of the proposed method applications are presented for the analysis of noise parameters of the fiber Raman amplifiers and for the generation bandwidth in fiber Raman lasers.

Keywords: optical amplification, Raman gain, fiber Raman lasers, fiber Raman amplifiers.
\end{abstract}

\section{Introduction}

Modern advances in the fiber lasers technology demonstrate the outstanding parameters such as the spectral purity and generation line width, output power, energy, and duration of ultrashort pulses [1]. They are primarily associated with the use of various doping impurities to silica glass, from which the most common fibers are made. Over the last decade, the impressive achievements in increasing the output power have been registered both for laser sources on rare earth ions [2-4] and for non-inverse lasers based on the effect of stimulated Raman scattering (SRS) of light $[5,6]$. For example, a fiber Raman laser (FRL) at an arbitrary wavelength can be built, by using a short $(\sim 30 \mathrm{~m})$ sample of standard single-mode fiber

(C) I.V. SERDEHA, V.I. GRYGORUK, G.S. FELINSKYI, 2018

ISSN 2071-0194. Ukr. J. Phys. 2018. Vol. 63, No. 8 made of silica glass [7]. However, the outstanding parameters of fiber lasers are realized, first of all, by modifying the fiber core with appropriate impurities.

For quite a long time, germanosilicate fibers were mainly used as the most efficient active medium for FRLs [8-10]. Just in germanosilicate fibers, the Raman cross-section of a core material is higher by more than one order of magnitude compared with fibers based on pure $\mathrm{SiO}_{2}$ [11]. Thus, these fibers are successfully used for both fiber Raman amplifiers (FRAs) of optical signals and for FRLs [12, 13]. Recently [14], an FRL with an output power of $188 \mathrm{~kW}$ with the use of the 70-m-long germanium fiber was reported. A high-efficiency Raman converter from $1080 \mathrm{~nm}$ to $1120 \mathrm{~nm}$ with a $\mathrm{GeO}_{2}$-doped fiber is a key element of such laser with the output power as high as kilowatts.

A detailed analysis of the threshold conditions and the working generation band of an FRL based 


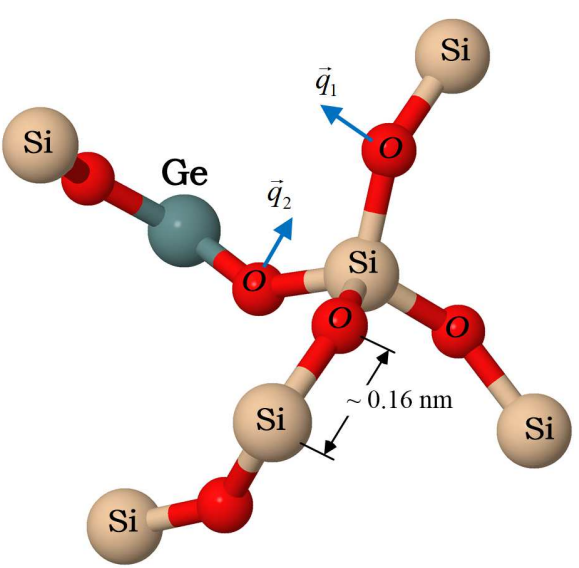

Fig. 1. Near order in the formation of amorphous glass from molecular nanocomplexes containing $\mathrm{Si}-\mathrm{O}-\mathrm{Si}$ and $\mathrm{Si}-\mathrm{O}-$ Ge bond types

on $\mathrm{GeO}_{2}$-doped single-mode fibers was presented in [15]. In the present paper, we describe our calculation technique and give the numerical results of the spectroscopic analysis of the Raman gain profile for a number of other single-mode fibers based on silica glass in the Stokes shift region from 0 to $1400 \mathrm{~cm}^{-1}$.

\section{Theoretical Basics \\ of Spontaneous and Stimulated Raman Scattering in Optical Fibers}

Spontaneous Raman scattering (RS) occurs as a result of the interaction between the optical wave field and vibrations of separate molecular nanocomplexes in amorphous compounds, from which the optical fiber core is made.

\subsection{Oscillatory model \\ of Raman active vibrations of molecular nanocomplexes in amorphous glass}

The most common fiber ("pure" $\mathrm{SiO}_{2}$ fiber) is made of silica glass $\mathrm{SiO}_{2}$ with small impurities of germanium $\mathrm{GeO}_{2}$ into the fiber core to form a waveguide. It should be noted that this is a standard fiber commonly referred to as a "pure" $\mathrm{SiO}_{2}$ fiber, but it actually contains several volume percents of $\mathrm{GeO}_{2}$ impurities inducing the formation of the region with increased refractive index. A change in the refractive index of a single-mode fiber core relative to the cladding makes up $\Delta n \sim 10^{-2}$, as a rule, and it directly depends on the $\mathrm{GeO}_{2}$ impurity concentration. At the same time, a small amount of germanium impurities makes it possible to attribute this most common type of fibers to pure $\mathrm{SiO}_{2}$ (hereinafter, the quotes symbols will be omitted). When the $\mathrm{GeO}_{2}$ concentration in the core is raised up to $25 \%$, the observed Raman gain coefficient is grown more than an order of magnitude in accordance with an increase in the stimulated Raman cross-section in the spectrum of molecular oscillation modes nearby the $440-\mathrm{cm}^{-1}$ peak. Because of a sharp increase in the Raman amplification, this type of fibers is referred to a separate class called a Raman fiber or $\mathrm{GeO}_{2}$-doped fiber, and it is used as a working active medium for the vast majority of modern Raman lasers.

The glass of both fiber cores is rather complicated, since it is formed by an amorphous compound from molecular nanocomplexes containing $\mathrm{Si}-\mathrm{O}-\mathrm{Si}, \mathrm{Si}-\mathrm{O}-$ Ge (Fig. 1), and seldom Ge-O-Ge bonds. We consider the elastic bond of an oxygen atom with two adjacent silicon or germanium atoms in the modeling of Raman processes. Since the oxygen atom is lighter than other atoms, it is often assumed [11] that the vibrations of these atoms create a harmonic oscillators system for the Raman process. Although the oscillating spectrum of molecular nanocomplexes is clearly not limited to oscillations of only oxygen atoms, it is efficient to quantitatively describe the features of the Raman gain against the background of the spontaneous scattering, by using the model of one oscillating mode.

In the most probable process of inelastic scattering of a photon of an optical radiation field on this oscillation, the oscillator energy is increased. Simultaneously with a decrease in the incident photon energy, the scattered light (Stokes wave) acquires a lower optical frequency, and its frequency is shifted by the phonon oscillation frequency of the mentioned molecular nanocomplexes. The spontaneous Raman crosssection does not depend on the intensity of excitation radiation and differs from zero in the centrally symmetric glass only for non-polar oscillations.

The relaxation of the excited oscillator to the future equilibrium position in irregular amorphous compounds, such as glass, occurs very rapidly (on femtosecond time intervals) through various interaction processes with other oscillators. Therefore, the renewal of the equilibrium state of glass occurs almost instantaneously. As a result, the spontaneous Raman cross-section turns out to be temperature-dependent due to the Bose factor of the phonon population on oscillator's energy levels.

ISSN 2071-0194. Ukr. J. Phys. 2018. Vol. 63, No. 8 
In the presence of a Stokes wave, which can be an external optical signal, the stimulated Raman process occurs. It becomes apparent in the exponential growth of the Stokes wave power propagating through the fiber. The conversion efficiency of the pumping power into a Stokes wave due to the Raman amplification is proportional to the pumping wave intensity. Therefore, in order to characterize the dynamics of the Raman gain process, the gain coefficient of the electromagnetic wave propagating in the fiber instead of the scattering cross-section is introduced.

Within our work, we rely on results of the stationary Raman theory in fibers for the quantum and semiclassical approaches. The Raman amplification process is described by means of continuous waves for both the pumping wave and signal wave. This approximation involving the rapid relaxation of fiber's phonon system is proved to be rightful for the experimental data interpretation even for relatively short pulses with subpicosecond duration and for signal frequencies extending up to several dozen GHz.

\subsection{Quantum dynamics of spontaneous and stimulated light scattering on phonons}

In terms of quantum dynamics, the elementary act of Raman scattering consists in the that a photon with angular frequency $\omega_{p}$ is inelastically scattered on a molecule, and two events can occur: i) Stokes and ii) anti-Stokes processes. The Stokes process consists in the birth of a phonon with angular frequency $\omega_{v}$ in addition to a photon with a lower angular frequency $\omega_{s}=\omega_{p}-\omega_{v}$. In the case of anti-Stokes process, an incident photon receives the phonon energy and, after the scattering process, acquires a higher angular frequency $\omega_{a s}=\omega_{p}+\omega_{v}$. These events may occur spontaneously or be stimulated by photons at the Stokes or anti-Stokes frequencies.

Based on the quantum approach, the dynamic equation for a change in the number of photons $n_{s}$ with the frequency $\omega_{s}$ in the process of inelastic light scattering (spontaneous + stimulated) depending on the photon propagation $z$-distance along the fiber was obtained [16] in the first-order perturbation theory in the form

$\frac{d n_{s}}{d z}=A\left[n_{s} n_{p}-n_{s} n_{v}+\left(n_{v}+1\right) n_{p}\right]$

where $n_{p}$ is the number of pumping photons, $n_{v}$ is the number of phonons of molecular oscillations of a fiber core. Due to the fast relaxation of molecular oscillations, we can assume that $n_{v}$ is maintained under conditions of thermodynamic equilibrium, i.e., the population $n_{v}$ is determined by the Bose-Einstein distribution:

$n_{v}=\left[\exp \left(\hbar \omega_{v} / k_{\mathrm{B}} T\right)-1\right]^{-1}$,

where $\hbar=h / 2 \pi$ is the Planck constant, $k_{\mathrm{B}}$ is the Boltzmann constant, $T$ is the absolute temperature, and the coefficient $A$ in Eq. (1) has the form

$A=\left|\frac{\partial \alpha}{\partial q}\right|^{2} \frac{\pi \hbar^{2}}{4 V^{2} N m \varepsilon_{s} \varepsilon_{p} v} \rho\left(\hbar \omega_{f}\right) \frac{\omega_{p} \omega_{s}}{\omega_{v}}$,

where $\partial \alpha / \partial q$ is the differential polarizability of a molecule determined by a derivative of the polarizability $\alpha$ with respect to the displacement coordinate $q, m$ is the effective mass associated with a vibration, $N$ is the number of oscillators in the interaction volume $V, \varepsilon_{s}$ and $\varepsilon_{p}$ are the dielectric permittivities for Stokes and pumping waves, correspondingly, they are given in scalar approximation; $v=c / \sqrt{\varepsilon_{s} / \varepsilon_{0}}$ is the phase velocity of a Stokes wave, $c$ is the light velocity in vacuum, $\varepsilon_{0}$ is the dielectric constant, and $\rho\left(\hbar \omega_{f}\right)$ is the density of final states, in which the system may find itself after the scattering. The density involves the sum of contributions from all possible final states.

The density of states $\rho\left(\hbar \omega_{f}\right)$ is a parameter of quantum theory. It is difficult to calculate the density of states directly, especially in the case of the irregular medium of an amorphous fiber core, since it requires the determination of all electron-vibrational energy levels of each molecule. In addition, this density depends on the phonon damping, which returns the system from the final state to the initial one through molecular transitions. There are many processes that return the system to a state of thermal equilibrium.

Despite the difficulties associated with the exact solution of the quantum equation (1), we can make a number of important conclusions regarding the physical features of the Raman amplification of a Stokes photon against the background of spontaneous Raman scattering as the main source of Stokes noise in the processes of nonlinear Raman amplification of optical radiation. The first positive term $n_{s} n_{p}$ in the square brackets on the right-hand side of Eq. (1) is responsible for the exponential growth of Stokes photons due to the stimulated Raman effect in the process of propagation of photons along the $z$-direction 
of the fiber. The second term, $-n_{s} n_{v}$, describes the own attenuation of Stokes photons. Finally, the third term, $\left(n_{v}+1\right) n_{p}$, describes the spontaneous Raman scattering, since with a given pumping, $n_{p}=$ const, the number of Stokes photons does not change at any fixed length $l$ of the fiber. For the next data analysis, it is necessary to specify two differences between the processes of spontaneous and stimulated Raman scatterings.

First, the stimulated radiation intensity does not depend on the temperature in contrast to the spontaneous radiation, for which the factor $n_{v}+1$ has essential temperature dependence in accordance with Eq. (2). Second, the occurrence of the stimulated Raman scattering has a threshold nature. Let the number of pumping photons, $n_{p} \ll n_{v}$, be small. Then the stimulated Raman scattering can be neglected. According to (1), $d n_{s} / d z<0$, i.e., Stokes photons are absorbed during the propagation process. The attenuation of Stokes waves stops at $n_{p}=$ $=n_{v}$. Therefore, the stimulated Raman scattering will be noticeable over the spontaneous Stokes noise background at $n_{p}>n_{v}$.

\subsection{Classical electrodynamics of light scattering processes in single-mode fibers}

According to classical calculations, whose results are given in this subsection, the Lorentz-type form of a Raman line appears quite naturally. Therefore, classical electrodynamics under conditions of the homogeneous broadening of vibrational energy levels in a system containing $N$ oscillators actually substantiates the use of a Lorentz lineform for the quantum mechanical value $\rho\left(\hbar \omega_{f}\right)$. Moreover, in amorphous fibers, where the orientation of a molecular nanocomplex relative to neighbor's environment is wholly random, the broadening of an oscillatory level becomes heterogeneous, and the oscillatory level can acquire the Gaussian form.

In order to describe the Raman scattering in an optical fiber in terms of classical electrodynamics, the analysis is advisable to limit by the one-mode field scattering. The full electric field in the case of Raman gain should be considered as the sum of two monochromatic waves, one at the pump frequency $\omega_{p}$, and the other at the scattered wave frequency $\omega_{s}$, which coincides, under the amplification, with the signal frequency. Next, the corresponding indices $s$ and $p$ are used to indicate the signal and pumping waves. If we restrict ourselves to the most common types of fibers in our consideration, namely, to those with weak waveguide properties, then it is possible to divide the electric field into the transverse part $R^{i}(r)$, (where $i=s, p$, and $r=\sqrt{x^{2}+y^{2}}$ ) and the function of $z: E_{i}(z)$. Then the full electric field takes the form

$\mathbf{E}=\mathbf{e}^{p} E^{p}(z) R^{p}(r) \exp \left\{i\left(\beta_{p} z-\omega_{p} t\right)\right\}+$

$+\mathbf{e}^{s} E^{s}(z) R^{s}(r) \exp \left\{i\left(\beta_{s} z-\omega_{s} t\right)\right\}+$ c.c.,

where $\mathbf{e}^{i}$ is a unit polarization vector, and $\beta_{i}$ is the propagation constant. It is determined, by solving the eigenvalue problem of a waveguide $\left[\nabla_{\perp}^{2}+\right.$ $\left.+\left(\varepsilon_{i} / \varepsilon_{0}\right)\left(\omega_{i}^{2} / c^{2}\right)\right] R^{i}=\beta_{i}^{2} R^{i}$, where $\nabla_{\perp}^{2}$ is the two-dimensional Laplace operator. The abbreviation c.c. in Eq. (3) denotes a complex conjugation of the previous expression.

The polarization induced by the electric field is equal to the density of dipole moments and has the form

$P_{i}=P_{i}^{L}+P_{i}^{N L}=n_{0} \xi_{i j}^{0} E_{j}+n_{0} \frac{\partial \xi_{i j}}{\partial q_{k}} q_{k} E_{j}=$

$=\alpha_{i j}^{0} E_{j}+\frac{\partial \alpha_{i j}}{\partial q_{k}} q_{k} E_{j}$,

where $n_{0}=N / V$ is the concentration of molecules, the vector $\mathbf{q}$ describes a displacement of the oscillating mass in a molecule from the equilibrium position, $\alpha_{i j}=n_{0} \xi_{i j}$ is the macroscopic polarizability of the scattering medium, which is the sum of all dielectric susceptibilities $\xi_{i j}$ of individual molecules in the unit volume, and the sum by repeated indices is implicitly assumed in Eq. (4) and so forth. The first term on the right-hand side describes the Rayleigh scattering, the second term describes the Raman scattering, and the tensor $\alpha_{i j k}=\partial \alpha_{i j} / \partial q_{k}$ is the Raman tensor. In terms related to the description of non-linear optical phenomena, $P_{i}^{L}=\chi_{i j}^{(1)} E_{j}$ and $\chi_{i j}^{(1)} \equiv \alpha_{i j}^{0}$. In the case of isotropic core of an optical fiber, as well as for other media with an inversion center, we have $\chi_{i j k}^{(2)} \equiv 0$. Therefore, at the description of the stimulated Raman process in fibers, the nonlinear polarization should have the form

$P_{i}^{N L}=\varepsilon_{0} \chi_{i j k l}^{(3)} E_{j} E_{k} E_{l}$.

The relationship between the nonlinear susceptibility of the third order $\chi_{i j k l}^{(3)}$ and the Raman tensor

ISSN 2071-0194. Ukr. J. Phys. 2018. Vol. 63, No. 8 
$\alpha_{i j k}=\partial \alpha_{i j} / \partial q_{k}$ may be installed as follows. The vector $\mathbf{q}$ depends on $\mathbf{E}$ in the form (3) for the case of stimulated Raman effect. The synchronous external force $\mathbf{F}$ is created by a corresponding combination of pumping fields and the Stokes wave, and the resonant behavior of this oscillation is excited. This vector $\mathbf{q}$ is described by the equation of a forced harmonic oscillator

$\frac{d^{2} \mathbf{q}}{d t^{2}}+2 \Gamma \frac{d \mathbf{q}}{d t}+\Omega_{0 v}^{2} \mathbf{q}=\frac{\mathbf{F}}{m}$,

where $\Gamma$ is the damping constant, and $\Omega_{0 v}$ is the selfresonant frequency of a harmonic oscillator without attenuation, $m$ is the effective mass of the oscillation. Note that the homogeneous equation in (6), as is known [17], must have quasiperiodic solutions only in the case of weakly damped oscillations $\Gamma<\Omega_{0 v}$, and its resonance frequency is equal to $\Omega_{v}=\sqrt{\Omega_{0 v}^{2}-\Gamma^{2}}$, i.e. it decreases under the attenuation influence. To solve Eq. (6), the shift $\mathbf{q}$ is expressed as

$\mathbf{q}=\mathbf{Q} e^{i\left(\beta_{v} z-\omega_{v} t\right)}+$ c.c.,

where $\beta_{v}$ and $\omega_{v}$ are, respectively, the wave number and the current frequency of forced oscillations of the damped phonon mode. As the Raman active vibration is dipole-inactive in the case of isotropic medium of the fiber core, the components of the external force vector $F_{k}$ should have the form [16]:

$F_{k}=\frac{1}{2} \frac{\partial \alpha_{i j}}{\partial q_{k}} E_{j} E_{i}$

The resonance of stimulated oscillations for the fields in the form (3) will be realized to obey the synchronization conditions as a result of the energy and momentum conservation laws:

$$
\begin{aligned}
& \omega_{p}=\omega_{s}+\omega_{v}, \\
& \beta_{p}=\beta_{s}+\beta_{v} .
\end{aligned}
$$

Leaving only the synchronous components for the force in (8) in the product of fields as in (3), we obtain

$F_{n}=\frac{1}{2} R^{p}\left(R^{s}\right)^{*} \frac{\partial \alpha_{k l}}{\partial q_{n}} E_{k}^{p}\left(E_{l}^{s}\right)^{*} e^{i\left(\beta_{v} z-\omega_{v} t\right)}+$ c.c. $=$

$=F_{0} e^{i\left(\beta_{v} z-\omega_{v} t\right)}+$ c.c.,

which allows us to write solution (6) in the form

$\mathbf{Q}=\frac{\mathbf{F}_{0}}{m} \frac{1}{\left(\Omega_{v}^{2}-\omega_{v}^{2}\right)-\left(2 i \omega_{v} \Gamma\right)}+$ c.c.
The induced polarization at the frequency $\omega_{s}$ may be expressed from Eqs. (3) and (4) in two equivalent forms as the amplitude at the oscillatory term $\exp \left\{i\left(\beta_{s} z-\omega_{s} t\right)\right\}$ in the form

$$
\begin{aligned}
& P_{i}^{\omega_{s}}=\frac{N}{2 m V} \frac{R^{s}\left|R^{p}\right|^{2}}{\left(\Omega_{v}^{2}-\omega_{v}^{2}\right)+\left(2 i \omega_{v} \gamma\right)} \times \\
& \times\left[\frac{\partial \alpha_{i j}}{\partial q_{n}}\left(\frac{\partial \alpha_{k l}}{\partial q_{n}} E_{k}^{p}\right)^{*} E_{l}^{s} E_{j}^{p}\right]= \\
& =6 \varepsilon_{0}\left|R^{p}\right|^{2} R^{s}\left[\chi_{i j k l}^{(3)} E_{j}^{p}\left(E_{k}^{p}\right)^{*} E_{l}^{s}\right]
\end{aligned}
$$

with the complex conjugation at the frequency $-\omega_{s}$. Thus, the expression for the susceptibility tensor of the third-order $\chi^{(3)}$ is:

$$
\begin{aligned}
& \chi_{i j k l}^{(3)}=\frac{N}{12 m \varepsilon_{0} V} \frac{1}{\left(\Omega_{v}^{2}-\omega_{v}^{2}\right)+\left(2 i \omega_{v} \Gamma\right)} \times \\
& \times \sum_{n} \frac{\partial \alpha_{i j}}{\partial q_{n}}\left(\frac{\partial \alpha_{k l}}{\partial q_{n}}\right)^{*},
\end{aligned}
$$

or

$\chi_{i j k l}^{(3)}=\frac{b_{i j k l}}{\left(\Omega_{v}^{2}-\omega_{v}^{2}\right)+\left(2 i \omega_{v} \Gamma\right)}=b_{i j k l} \cdot \tilde{\varphi}\left(\omega_{v}\right)$,

where the tensor components are

$b_{i j k l}=\frac{N}{12 m \varepsilon_{0} V} \sum_{n} \frac{\partial \alpha_{i j}}{\partial q_{n}}\left(\frac{\partial \alpha_{k l}}{\partial q_{n}}\right)^{*}$.

They do not depend on the Stokes shift frequency $\omega_{v}$, as distinct from the complex function $\tilde{\varphi}\left(\omega_{v}\right)$ describing the resonant behavior of the third-order susceptibility $\chi^{(3)}$ near own frequencies $\Omega_{v}$ of a molecule oscillations.

Thus, Eq. (12) gives the quantitative expression of the nonlinear relationship between the Stokes wave and the pumping wave, when they are simultaneously propagating along the $z$-direction of the fiber. Then the amplitude of the Stokes electric field $\mathbf{E}=\mathbf{E}^{s}=$ $=\mathbf{e}^{s} E^{s}(z) R^{s}(r) \exp \left\{i\left(\beta_{s} z-\omega_{s} t\right)\right\}$ will be slowly changing in the $z$-direction due to the smallness of components $\chi_{i j k l}^{(3)}$ and $\alpha_{i j k}=\partial \alpha_{i j} / \partial q_{k}$. That is why the term $\partial^{2} \mathbf{E}^{s} / \partial z^{2}$ may be neglected, and the wave equation for the amplitudes of each Stokes component of scattered waves may be written as

$\frac{\partial E_{i}^{s}}{\partial z} R^{s}=\frac{3 \omega_{s}^{2}}{c^{2} \beta_{s}} i R^{s}\left|R^{p}\right|^{2}\left[\chi_{i j k l}^{(3)} E_{j}^{p}\left(E_{k}^{p}\right)^{*} E_{l}^{s}\right]$. 


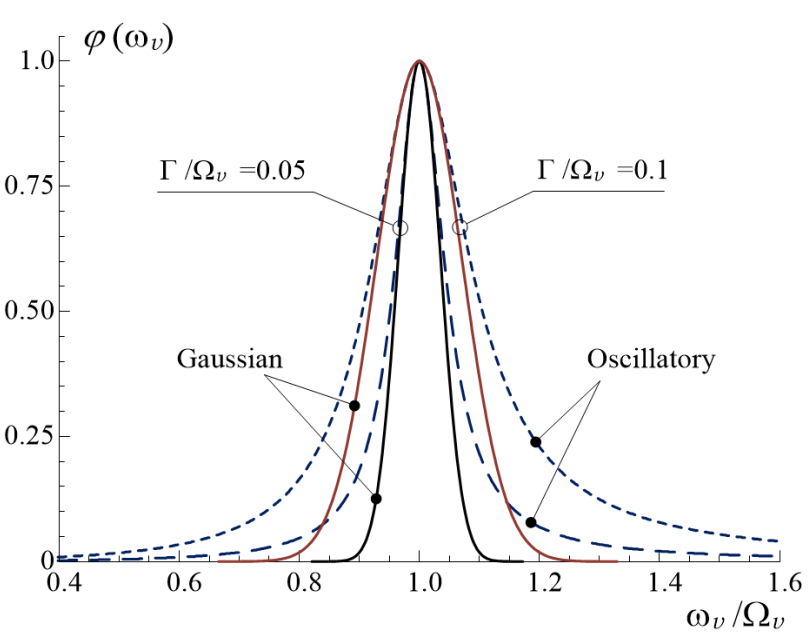

Fig. 2. Normalized form-factors for Raman gain profiles: Gaussian profile (continuous line) and profile of a damped oscillator (dotted line) for $\Gamma / \Omega_{v}=0.05$ and $\Gamma / \Omega_{v}=0.1$

In practice, the most important is the dynamic equation for the power $P^{s}=2 \varepsilon_{0} n_{s} c \int_{A}\left|E^{s} E^{p}\right|^{2} d A$, where $A$ is the cross-section area of a fiber. It can be obtained directly from (16) in the form [10]

$\frac{d P^{s}}{d z}=\gamma_{R} P^{s}=g_{R} P^{p} P^{s}$

This expression describes the exponential Raman amplification of a Stokes wave in single-mode fibers.

\subsection{Spectral Raman gain profile}

The gain coefficient of the Stokes wave power $\gamma_{R}$ occurs to be directly proportional to the pumping power $\gamma_{R}=g_{R} P^{p}$, whereas the gain coefficient $g_{R}$ depends only on the fiber parameters in the form

$g_{R}=-\frac{3 \omega_{s}^{2}}{\varepsilon_{0} n_{p} c^{3} \beta_{s}} \frac{1}{A_{\mathrm{eff}}^{p s}} \frac{\operatorname{Im}\left[\chi_{i i i i}^{(3)}+\chi_{i j j i}^{(3)}\right]}{2}$,

where $A_{\text {eff }}^{p s}$ is the effective area of the overlap of the pumping and the signal waves

$A_{\mathrm{eff}}^{p s}=\frac{\int_{A}\left|R^{p}\right|^{2} d A \int_{A}\left|R^{s}\right|^{2} d A}{\int_{A}\left|R^{p}\right|^{2}\left|R^{s}\right|^{2} d A}$.

The Raman gain coefficient $g_{R}$ is a function of the Stokes shift frequency $\omega_{v}$. If we assume that the Raman tensor components are real numbers $\alpha_{i j k}=\alpha_{i j k}^{*}$, then, according to (14) and (18), this dependence can be represented explicitly as

$$
g_{R}\left(\omega_{v}\right)=g_{R \max } \frac{4 \omega_{v}^{2} \Gamma^{2}}{\left(\Omega_{v}^{2}-\omega_{v}^{2}\right)^{2}+4 \omega_{v}^{2} \Gamma^{2}}=g_{R \max } \varphi\left(\omega_{v}\right) .
$$

The function $g_{R}\left(\omega_{v}\right)$ is called the Raman gain profile. Profile (20) has a resonant dependence of the damped oscillator (Lorentz type), since it is obtained in the one-oscillator model of an individual molecule. All oscillatory energy levels in the ensemble of oscillators are expanded, and the form-factor $\varphi\left(\omega_{v}\right)$ may remain as $(20)$ in the case of homogeneous broadening. With the random distribution of molecular movement orientations relative to the surroundings, the broadening of vibrational levels becomes heterogeneous. The form-factor $\varphi\left(\omega_{v}\right)$ takes the form

$\varphi\left(\omega_{v}\right)=\exp \left[-\frac{\left(\Omega_{v}-\omega_{v}\right)^{2}}{\Gamma^{2}}\right]$

and belongs the Gaussian type. Both profiles (20) and (21) are presented in Fig. 2 for two values of relative phonon's damping, namely for $\Gamma / \Omega_{v}=0.05$ and 0.1 . The broadening is mostly heterogeneous in such amorphous materials as glass. Below, we continue to use the Gaussian components (21) in order to decompose the complex Raman spectra in optical fibers.

\subsection{Raman gain features and its relation to the spontaneous scattering}

In fact, the difference between the Raman spectrum and the frequency Raman gain profile in single-mode silica fibers was found by the analysis of the quantum dynamic equation (1). Since the energy of $n$ photons with frequency $\omega$ in its numerical expression is equal to the spectral power density, the number $n$ actually determines the monochromatic radiation power in the unit frequency interval around $\omega$. Due to the quantum approach, the monochromatic power is interpreted as the number of photons in one longitudinal mode of optical radiation. This allows us to connect the Raman gain profile to the spontaneous Raman cross-section based on the dynamics of Stokes and pumping photons.

First of all, the absence of the temperature dependence of the Raman amplification makes the connection of the frequency profile $g_{R}\left(\omega_{v}\right)$ with the spontaneous cross-section such that the cross-section $\sigma_{0}(\omega)$ 
at the zero temperature corresponds to the Raman gain value $[18,19]$. So, $g_{R}\left(\omega_{v}\right)$ takes the form

$g_{R}\left(\omega_{v}\right)=\sigma_{0}\left(\omega_{v}\right) \frac{\lambda_{s}^{3}}{c^{2} h A_{\mathrm{eff}}^{p s} n_{p}^{2}}$,

where $\lambda_{s}$ is the Stokes wavelength, $c$ is the speed of light, $h$ is Planck's constant, and the weak frequency dependence of the refractive index $n_{p}$ in the frequency region of the Stokes shift can be neglected. The Raman cross-section $\sigma_{T}\left(\omega_{v}\right)$ measured at a temperature $T$ is referred to a zero-Kelvin cross-section $\sigma_{0}\left(\omega_{v}\right)$ as

$\sigma_{0}\left(\omega_{v}\right)=\sigma_{T}\left(\omega_{v}\right) /\left[n_{\mathrm{B}}\left(\omega_{v}, T\right)+1\right]$,

where $n_{B}\left(\omega_{v}, T\right)$ is the Bose-Einstein factor (2).

Another difference between the stimulated and spontaneous Raman processes is the amplification of a coherent Stokes wave during the stimulated Raman process, whereas the entire Stokes shift region corresponds to the incoherent optical noise as the spontaneous process. Experimental studies of the noise statistics of the amplified spontaneous emission (ASE) were carried out in [20] and confirmed the Bose-Einstein distribution for the noise appeared as ASE in the fiber Raman amplifiers.

Figure 3 shows the scattering cross-section $\sigma_{T}\left(\omega_{v}\right)$ in the spontaneous Raman spectra as a function of the temperature $T$, where the experimentally measured Raman gain profile is selected for the zeroKelvin cross-section $\sigma_{0}\left(\omega_{v}\right)$ in the most conventional fiber based on pure silica $\mathrm{SiO}_{2}$. Some idealization of the picture in Fig. 3 consists in the fact that the basic profile obtained for $T=300 \mathrm{~K}$ was considered unchanged over the whole temperatures range from $77 \mathrm{~K}$ to $500 \mathrm{~K}$.

In practice, the description is complicated by the fact that noticeable changes in the profile can occur both due to a change in the damping constant $\Gamma$ and a shift of the resonant frequencies $\Omega_{v}$ of a damped oscillator of molecules in the fiber core. As a result of a great decrease in the temperature, the damping $\Gamma$ decreases, as a rule, by several times as well, which leads to the greater separation of individual phonon modes of the oscillatory spectrum. According to $(6)$, the frequency shift $\Omega_{v}=\sqrt{\Omega_{0 v}^{2}-\Gamma^{2}}$ with respect to the own frequency of a non-damped oscillator $\Omega_{0 v}$ will be negligible with $\Gamma \ll \Omega_{0 v}$. However, the shift will be noticeable for highly damped lowfrequency oscillations. For $\Gamma>\Omega_{0 v}$, the oscillation

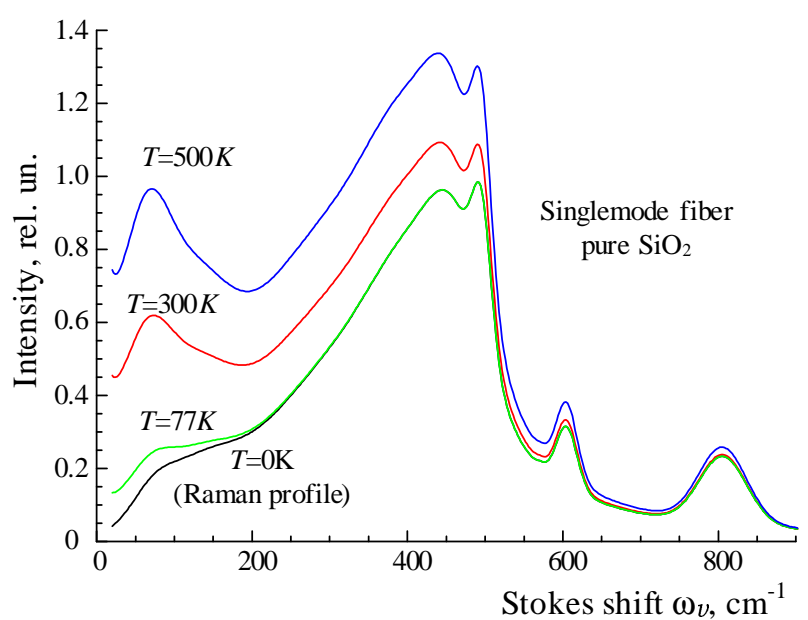

Fig. 3. Cross-section in the spontaneous Raman spectra as a function of the temperature and the Raman gain profile corresponding to the zero-Kelvin cross-section $\sigma_{0}\left(\omega_{v}\right)$

completely disappears from the phonon spectrum. At the same time, the difference between the spectra of the Raman scattering and the Raman amplification shown in Fig. 3 is observed experimentally at least in a certain proximity to room temperatures (it was observed in our investigations as well), when the pumping power does not exceed several hundred $\mathrm{mW}$. Note that such pumping regimes for the single-mode fiber are nominal for optical FRA, and they are close to the lasing threshold in FRL.

According to (22), (23), and the data shown in Fig. 3, the significant difference between the spontaneous Raman spectra and the Raman gain profile in pure $\mathrm{SiO}_{2}$ fibers, in particular at $T=300 \mathrm{~K}$, should be observed only in the frequency range of the Stokes shift less than $500 \mathrm{~cm}^{-1}$, where the thermal population factor of phonons is considerably greater than 1. The thermal population factor for Stokes phonons in the higher-frequency region $\left(>500 \mathrm{~cm}^{-1}\right)$ loses its frequency dependence and does not differ practically from 1 . Therefore, the spontaneous Raman spectrum coincides with the Raman gain profile.

Thus, the multiple growth of the cross-section $\sigma_{T}\left(\omega_{v}\right)$ for Stokes shifts less than $200 \mathrm{~cm}^{-1}$ relative to the Raman gain allows one to even visually detect the spontaneous Raman scattering, i.e. the incoherent Stokes noise in the optical spectrum of a silica fiber. On the other hand, this feature of the Raman processes was considered by us as the basis for the method to determine Raman gain profiles by the 
spontaneous Raman spectra in other types of fibers mainly based on silica glass.

\subsection{Absolute transparency and the lasing threshold due to a Raman gain in optical fibers}

To determine the Raman gain threshold, it is sufficient to consider the simplest case of the interaction between one pumping wave and one Stokes wave. Then the intensities of both waves in the quasicontinuous approximation with regard for the slowly changing wave amplitudes during their propagation along the fiber ( $z$ coordinate) are described by the system of two coupled equations:

$$
\begin{aligned}
& \frac{d I_{s}\left(z, \omega_{s}\right)}{d z}=\tilde{g}_{R}\left(\omega_{v}\right) I_{p}(z) I_{s}\left(z, \omega_{s}\right)-\alpha_{s} I_{s}\left(z, \omega_{s}\right), \\
& \frac{d I_{p}(z)}{d z}=\frac{\omega_{p}}{\omega_{s}} \tilde{g}_{R}\left(\omega_{v}\right) I_{p}(z) I_{s}\left(z, \omega_{s}\right)-\alpha_{p} I_{p}(z),
\end{aligned}
$$

where $\alpha_{s}$ and $\alpha_{p}$ take the fiber losses at the Stokes and pumping frequencies into account, respectively; $\tilde{g}_{R}\left(\omega_{v}\right)$ is the Raman gain coefficient, $I_{p}$ is the pump intensity, and $I_{s}$ is the Stokes wave intensity.

Equations (24) and (25) follow from the Maxwell equations and are derived analogously to Eq. (17). It should be noted that the variable frequency $\omega$ of both interacting waves is included in Eqs. (24) and (25) as a parameter. For the frequency dependences of the variable functions and coefficients in these equations, we apply the following approximations. The width of the pumping line can be considered infinitely narrow compared to the Stokes radiation band. In other words, the pump remains localized at its frequency $\omega_{p}$, and $I_{p}(z, \omega)=\left.I_{p}(z, \omega)\right|_{\omega=\omega_{p}}=I_{p}(z)$ and does not depend on the frequency at any point $z$ along the fiber. In fact, the system of equations (24), (25) describes the Raman interaction of monochromatic pumping waves and Stokes radiation.

Naturally, the wave coupling degree at any given frequency $\omega$ within the irregular continuum of Stokes shifted frequencies is unambiguously determined by the values of the functions $g_{R}(\omega)=\left.g_{R \max } \varphi(\omega)\right|_{\omega=\omega_{v}}$ considering the attenuation of both waves. It is determined by absorption coefficients $\alpha_{s, p}=\alpha_{s, p}(\omega)$. The function $g_{R}(\omega)$ is shown in Fig. 3 at $T=0$ for a single-mode pure $\mathrm{SiO}_{2}$ fiber, and it is a subject of the separate research in our work for other types of a fiber. The dependence $\alpha_{s, p}(\omega)$, as a rule, is rather weak. From this viewpoint, we consider the absorption coefficients $\alpha_{s}$ and $\alpha_{p}$ to be constant and frequency-independent in Eqs. (24) and (25).

From Eq. (24), the condition of absolute transparency of the fiber is directly resulted. Its physical sense corresponds to the lasing threshold of the Raman gain process. Since the pumping power $P_{p}(\omega)$ may be expressed in terms of the pump intensity $I_{p}(\omega)$ and the fiber effective area $A_{\text {eff }}$, the equality $d I_{s} / d z=0$ in view of the inequality $d P_{s}(\omega) / d z>0$ gives the following quantitative expression [15]

$P_{p}^{\mathrm{th}}=\frac{\alpha_{s} A_{\mathrm{eff}}}{\tilde{g}_{R}(\omega)}=\frac{\alpha_{s}}{g_{R}(\omega)}$,

where $\tilde{g}_{R}[\mathrm{~m} / \mathrm{W}]$ and $g_{R}[\mathrm{~W} \cdot \mathrm{km}]^{-1}$ are two equivalent forms of the Raman gain coefficients referred to the intensity $I_{p}$ and the power $P_{p}$, respectively. Expression (26) determines the spectral function of the full transparency of a fiber, i.e. $P_{p}^{\text {th }}=P_{p}^{\text {th }}(\omega)$ corresponds to the boundary condition when the material of the fiber core begins its transformation from the natural state with the Stokes wave attenuation to a state, in which the Stokes wave is amplified by the pumping power. For $P_{p}^{\text {th }}$, its minimum value at $g_{R}\left(\omega_{\max }\right)=g_{R \max }$ is usually chosen. The advantages of such definition of the amplification threshold are as follows.

Let the pumping power $P_{p}$ be known at a certain point $z$ of the fiber obtained as a result of the measurements or calculations. Then the direct verification of the inequality $P_{p}>(<) P_{p}^{\text {th }}$ with the known constant $\alpha$ and function $g_{R}(\omega)$ allow us to determine not only the frequencies, for which the condition of full transparency of the fiber is fulfilled, but also the amplification (generation) band that lies between them. A particular example of this modeling for a $\mathrm{TiO}_{2}$-doped fiber is given in Section 3 .

Thus, using Eq. (26) and available experimental data for the Raman gain profile $g_{R}(\omega)$, we can directly calculate the lasing threshold as a function of the frequency (or wavelength) in the region of Stokes shifted frequencies for an arbitrary wavelength of the pumping source. According to (20) and (21), the gain profile $g_{R}(\omega)$ of a particular oscillatory mode may be described by simple lineform functions, namely those of the oscillatory (Lorentz) type and/or Gaussian type. However, real fibers have such complex gain profile that the satisfactory shape of the function

ISSN 2071-0194. Ukr. J. Phys. 2018. Vol. 63, No. 8 


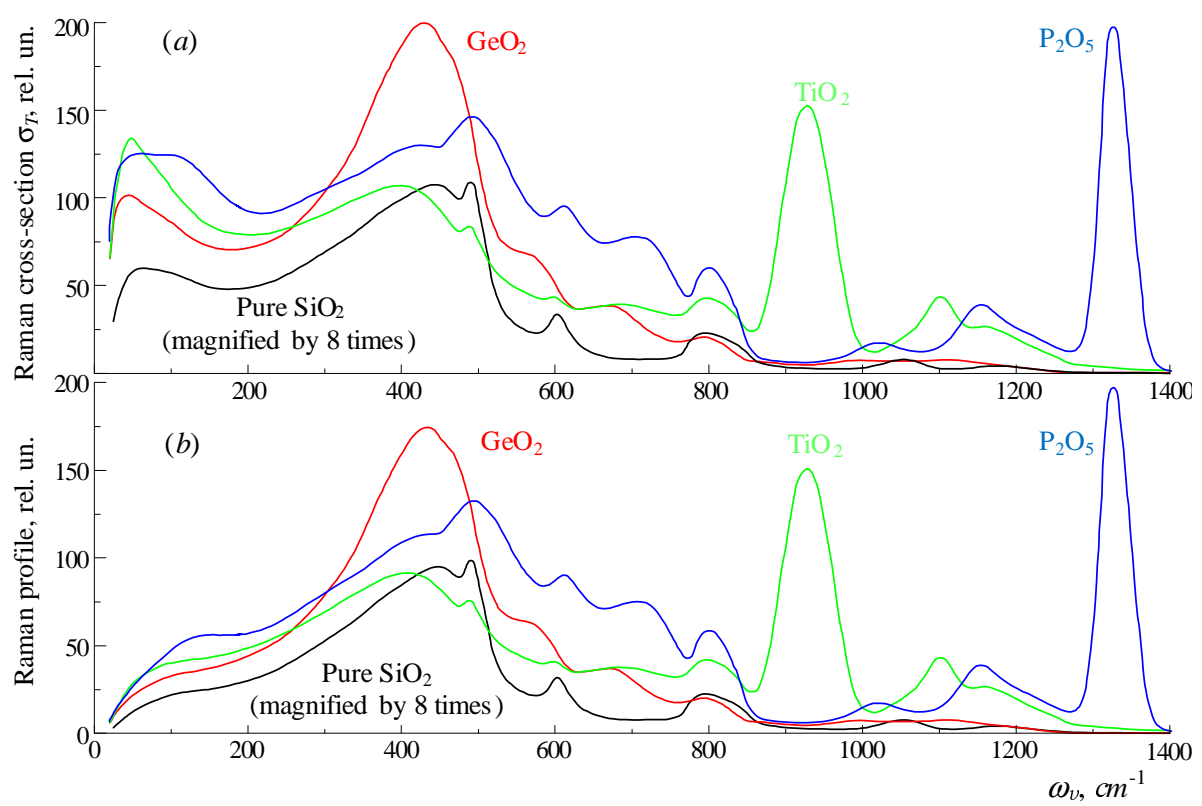

Fig. 4. Raman spectra $(a)$ and Raman gain profiles $(b)$ in single-mode fibers based on fused silica

$g_{R}(\omega)$ may be obtained by a special modeling. The multimode decomposition model of the Raman gain profile and the results of its application with the use of Gaussian-type components for a number of fibers are presented in the next section.

\section{Raman Gain in Single-Mode Fibers Based on Fused Silica}

Our analysis of Raman gain profiles is based on the experimental spectra of spontaneous Raman scattering. A general view of the Raman spectra is shown in Fig. 4, $a$ for single-mode fibers with different dopants in the core, namely for pure $\mathrm{SiO}_{2}$ fibers and for $\mathrm{GeO}_{2}$ and for $\mathrm{TiO}_{2}$-doped ones, as well as for phosphorussilicate fibers (with $\mathrm{P}_{2} \mathrm{O}_{5}$ impurities). The Raman spectra in all optical fibers based on silica glass are characterized by the appearance of an irregular continuum, which is formed due to the overlapping of strongly extended modes of the oscillating spectrum.

Because of the large difference in the scattering cross-sections, the spectrum of pure $\mathrm{SiO}_{2}$ in Fig. 4 is depicted for the convenience of comparison with other fiber spectra, in particular with that of a $\mathrm{GeO}_{2}$-doped fiber, on a scale increased by $\sim 8$ times. Both types of silica fibers are the most investigated, and their Raman gain coefficients are well known. So, they are used in our work to calibrate the absolute gain values in other types of silica fibers.

Other fiber types $\left(\mathrm{TiO}_{2}{ }^{-}\right.$and $\mathrm{P}_{2} \mathrm{O}_{5}$-doped fibers) are developed more later; they have good perspectives as an active medium for the nonlinear optical amplification, but they have been significantly less studied. The advantages of phosphorus-silicate fibers were demonstrated in [8] as an example of the cascade FRL generation, by using a Stokes shift of $1330 \mathrm{~cm}^{-1}$ in the molecular vibration spectrum of this fiber core. The field of applicability of these fibers can be expanded, by using of the entire area of Stokes shifts, in particular, the frequency range $\sim 500-1400 \mathrm{~cm}^{-1}$. In this paper, we analyze comprehensively the Raman gain profiles for these single-mode fibers in a wide frequency range of Stokes shifts.

The preliminary theoretical analysis of the Raman scattering processes forms the basis for the direct determination of a Raman gain profile from the experimental Raman spectrum as its part that does not depend on the temperature. Then we use the spectroscopic technique for determining the Raman gain profile in an analytic form, and it is based on the multimode decomposition of the complex spectrum in a definite region of Stokes shift frequencies.

Note that the amorphous nature of silica glass significantly complicates the solution of such rather 
standard spectroscopic problems in almost every case of optical fiber, and their general solutions have not been obtained yet. However, we succeeded to find a special solution to this problem in a number of individual cases, in particular, in the case of $\mathrm{TiO}_{2}$-doped fiber [21], [22]. In this paper, we also give an analytic expression for the Raman gain profile in a phosphoric silicate fiber, which has explicit prospects for the use as an active medium in modern devices of fiber nonlinear optics.

\subsection{Determination of Raman gain profiles by spontaneous spectra in silica fibers}

Our method for the determination of a Raman gain profile from the spontaneous spectra is based on formulas (22) and (23). It consists in the separation of the component, which corresponds to the zerotemperature (on the Kelvin scale) spectrum of own molecular oscillations from spontaneous spectra and does not depend on the absolute temperature of the fiber. The result of such processing of spontaneous spectra of all investigated fibers is presented in Fig. 4, $b$.

The Raman gain profiles are mainly different from the spontaneous Raman spectrum by a significant decrease in the absolute intensity of low-frequency components due to the spectral distribution of the Bose factor of the phonon population (2). Indeed, at the frequency corresponding to the wavenumber equal to $20 \mathrm{~cm}^{-1}$, the spectral density of spontaneous noise is by 11 times greater than the Raman gain at $T=300 \mathrm{~K}$. This difference between Raman gain profiles and the spontaneous spectra becomes $\sim 13$ $14 \%$ at the frequency $\sim 440 \mathrm{~cm}^{-1}$, where Raman gain coefficients reach their maximum for pure $\mathrm{SiO}_{2}$ and $\mathrm{GeO}_{2}$-doped fibers. Moreover, the ratio of the spectral density of the Stokes noise to the Raman amplification decreases below $1 \%$ level only at frequencies exceeding $880 \mathrm{~cm}^{-1}$ at room temperature. Therefore, the absolute maxima of the Raman gain coefficients for $\mathrm{TiO}_{2}$ - and $\mathrm{P}_{2} \mathrm{O}_{5}$-doped fibers located at frequencies of $\sim 930 \mathrm{~cm}^{-1}$ and $\sim 1330 \mathrm{~cm}^{-1}$, respectively, practically coincide with the values of the differential Raman cross-section at least for typical ambient temperatures $T \leq 300 \mathrm{~K}$.

The normalized Raman gain profiles separated from the experimental spontaneous spectra for the Stokes shift region of $0-1400 \mathrm{~cm}^{-1}(0-42 \mathrm{THz})$ are presented in Fig. 5 for SMF made from silica glass: $a$ - for pure $\mathrm{SiO}_{2} ; b, c$, and $d$ - for $\mathrm{GeO}_{2^{-}}, \mathrm{TiO}_{2-}$, and $\mathrm{P}_{2} \mathrm{O}_{5}$-doped fibers, respectively. The values of the gain coefficients at the maximum frequency points of the Raman profile were given earlier in [11], namely, $g_{R \max }=0.4(\mathrm{~W} \cdot \mathrm{km})^{-1}$ for pure $\mathrm{SiO}_{2}$ and $g_{R \max }=$ $=6.3(\mathrm{~W} \cdot \mathrm{km})^{-1}$ for specialized Raman fiber with the $\mathrm{GeO}_{2}$ dopant concentration in the core up to $25 \%$. We supplemented the data of work [11] by extending the Stokes shifts region up to $1400 \mathrm{~cm}^{-1}$, as is shown in Fig. 4, $a, b$.

The estimation of $g_{R \max }$ values for $\mathrm{TiO}_{2^{-}}$and $\mathrm{P}_{2} \mathrm{O}_{5}$-doped fibers was made by comparing the absolute intensities of main spectral components in the experimental Raman spectra, since they are unambiguously associated with the corresponding Raman gain profiles. According to this estimation, $g_{R \max }=$ $=4.8(\mathrm{~W} \cdot \mathrm{km})^{-1}$ is achieved at a Stokes shift frequency of $930 \mathrm{~cm}^{-1}$ for a $\mathrm{TiO}_{2}$-doped fiber. In $\mathrm{P}_{2} \mathrm{O}_{5-}$ doped fiber, the maximum value of the Raman gain coefficient reaches, according to data [9], 90\% of the gain in a $\mathrm{GeO}_{2}$-doped fiber and, therefore, should be equal to $g_{R \max }=5.7(\mathrm{~W} \cdot \mathrm{km})^{-1}$ at the Stokes shift frequency $\omega_{\max }=1330 \mathrm{~cm}^{-1}$.

The absolute value of $g_{R \text { max }}$ together with the normalized Raman gain profiles unambiguously determines the magnitude of the nonlinear signal gain at a given point of the Stokes shift band. Despite the given numerical values of $g_{R \max }$ can vary slightly, by depending on the technological specific features affecting the effective area of fibers, the presented estimates allows us to obtain a quantitative idea of the nonlinear-optical properties of the fiber core of the investigated silica glass and its chemical composition.

All Raman gain profiles presented in Fig. 5 can be conventionally divided into two almost equal spectral regions. The first one is from 0 to $700 \mathrm{~cm}^{-1}$. It contains the main maxima of the Raman gain from the base material of the fiber core, that is, the silica glass. The second region from $700 \mathrm{~cm}^{-1}$ to $1400 \mathrm{~cm}^{-1}$ can be assigned to the dopant oscillatory spectrum, since its small intensity of Raman bands can be observed in both pure $\mathrm{SiO}_{2}$ and in the standard fiber strongly doped with $\mathrm{GeO}_{2}$. In contrast, the bands containing the Raman gain maximum in two other samples of both $\mathrm{TiO}_{2}$ - and $\mathrm{P}_{2} \mathrm{O}_{5}$-doped fibers are located in the second (i.e., dopant) region. However, both impurities $\mathrm{TiO}_{2}$ and $\mathrm{P}_{2} \mathrm{O}_{5}$ simultaneously considerably perturb the phonon spectrum in the first 
low-frequency region as well. The absolute intensity of these molecular vibrations is increased by several times compared with pure $\mathrm{SiO}_{2}$.

Because of the irregularity of the quasicontinuous vibrational spectrum and the "diffuse" nature of Raman lines, whose width may exceed $100 \mathrm{~cm}^{-1}$ with a strong overlap in Raman spectra of silica glass, it is necessary to use special methods of spectroscopic modeling of Raman gain profiles. The simulation basis is the theory presented in Section 2. According to it, the Stokes shift frequencies in the Raman process are placed within the band of spontaneous Raman scattering from each of the oscillatory modes of the fiber core material.

\subsection{Multimode decomposition of Raman gain profiles}

The rationale of the multimode decomposition method for Raman gain profiles is proved by the several formation peculiarities of phonon spectra in different types of glass noted in many papers [9-11, 23, 24 . In particular, although the profile of the refractive index changes in the fiber core repeats the distribution of the dopant concentration, the Raman gain spectra of the germanosilicate fibers are not a simple mixture of the spectra of pure $\mathrm{SiO}_{2}$ and germanium glass $\mathrm{GeO}_{2}$ [11]. The essential deformations of Raman gain profiles in glass of one type relative to glass of another type are observed in practice. This indicates a significant change in all the vibration parameters, such us amplitude, frequency position, and damping constant. Moreover, the Raman gain coefficients of some germanosilicate composites can significantly exceed the values that have both pure components of this glass.

The significant expansion of oscillatory spectrum bands of molecular oscillations of more than $100 \mathrm{~cm}^{-1}$ is typical of the materials based on amorphous glass. So, the Stokes region in each fiber is converted into an irregular continuum (Figs. 3 and 4), where it is practically impossible to separate the frequencies of individual oscillating modes. The analysis of the spontaneous Raman spectra of pure $\mathrm{SiO}_{2}$ in the Stokes region between 0 and $900 \mathrm{~cm}^{-1}$ was presented in [23]. The authors found at least 10 separate components by the careful attachment to the observed spectral features such as the maxima positions, slopes, and pedestals available in the spectrum, and these components were classified as those of the Gaussian type.

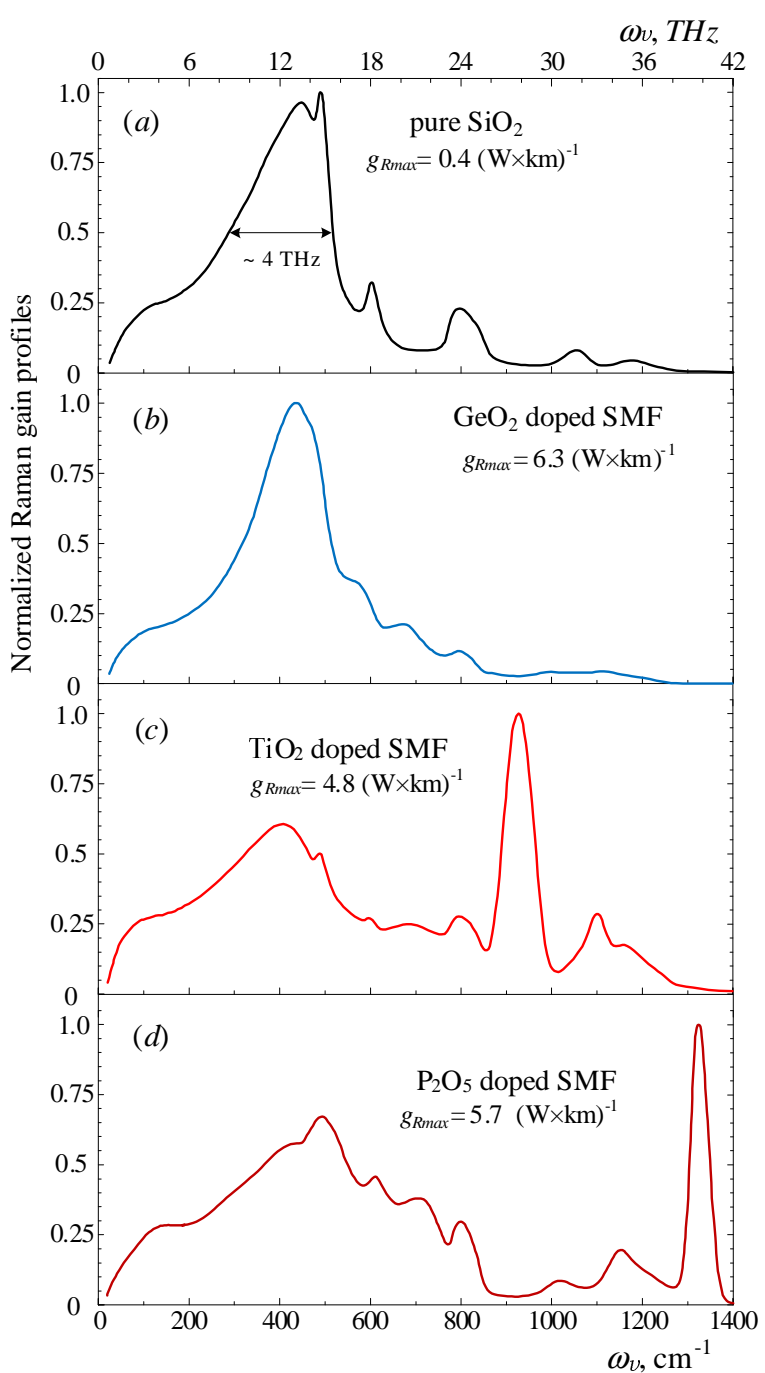

Fig. 5. Normalized Raman gain profiles of silica fibers in the Stokes shift region $\left.0-1400 \mathrm{~cm}^{-1}(0-42 \mathrm{THz})\right)$

In our work, the decomposition into Gaussian components in a pure $\mathrm{SiO}_{2}$ fiber core was carried out for Raman gain profiles in the Stokes shift region up to $1400 \mathrm{~cm}^{-1}$.

The oscillatory dynamics of molecular nanocomplexes of the amorphous fiber core underlies the formation of Raman gain profiles. So, the profiles can be modeled as a system of harmonic oscillators forced by the external rather powerful pumping wave. Some simplifications of the model consist in the neglect of the frequency dependence of the effective area $A_{\text {eff }}^{p s}$ and the refractive index $n_{p}$, as well as the dependence of the Raman gain coefficient $g_{R}$ on the Stokes 
Results of multimode decomposition of Raman gain profiles in silica fibers.

To estimate the approximation accuracy, the parameter $\delta=\left(I_{G}-I_{0}\right) / I_{0} \times 100 \%$ is presented, where $I_{G}$ and $I_{0}$ are the corresponding integral intensities of the calculated and measured Stokes spectra

\begin{tabular}{|c|c|c|c|c|c|c|c|c|c|c|c|c|}
\hline \multirow{2}{*}{ No. } & \multicolumn{3}{|c|}{ Pure $\mathrm{SiO}_{2}$} & \multicolumn{3}{|c|}{$\mathrm{GeO}_{2}$} & \multicolumn{3}{|c|}{$\mathrm{TiO}_{2}$} & \multicolumn{3}{|c|}{$\mathrm{P}_{2} \mathrm{O}_{5}$} \\
\hline & $A_{i}$ & $\begin{array}{c}\Omega_{v i} \\
\mathrm{~cm}^{-1}\end{array}$ & $\begin{array}{c}\Gamma_{i}, \\
\mathrm{~cm}^{-1}\end{array}$ & $A_{i}$ & $\begin{array}{c}\Omega_{v i} \\
\mathrm{~cm}^{-1}\end{array}$ & $\begin{array}{c}\Gamma_{i} \\
\mathrm{~cm}^{-1}\end{array}$ & $A_{i}$ & $\begin{array}{c}\Omega_{v i} \\
\mathrm{~cm}^{-1}\end{array}$ & $\begin{array}{c}\Gamma_{i} \\
\mathrm{~cm}^{-1}\end{array}$ & $A_{i}$ & $\begin{array}{c}\Omega_{v i} \\
\mathrm{~cm}^{-1}\end{array}$ & $\begin{array}{c}\Gamma_{i} \\
\mathrm{~cm}^{-1}\end{array}$ \\
\hline $\mathrm{G}_{1}$ & 0.07 & 71 & 39 & 0.04 & 48 & 25 & 0.11 & 67 & 38 & 0.05 & 72 & 35 \\
\hline $\mathrm{G}_{2}$ & 0.12 & 128 & 67 & 0.09 & 89 & 53 & 0.13 & 130 & 75 & 0.13 & 131 & 67 \\
\hline $\mathrm{G}_{3}$ & 0.54 & 359 & 182 & 0.18 & 179 & 102 & 0.53 & 381 & 215 & 0.04 & 410 & 31 \\
\hline $\mathrm{G}_{4}$ & 0.25 & 408 & 68 & 0.49 & 360 & 119 & 0.09 & 418 & 56 & 0.53 & 437 & 268 \\
\hline $\mathrm{G}_{5}$ & 0.35 & 465 & 49 & 0.68 & 448 & 80 & 0.07 & 493 & 14 & 0.17 & 502 & 47 \\
\hline $\mathrm{G}_{6}$ & 0.32 & 496 & 17 & 0.08 & 481 & 21 & 0.03 & 599 & 16 & 0.11 & 618 & 31 \\
\hline $\mathrm{G}_{7}$ & 0.01 & 571 & 8 & 0.25 & 573 & 43 & 0.19 & 712 & 94 & 0.21 & 716 & 55 \\
\hline $\mathrm{G}_{8}$ & 0.16 & 605 & 19 & 0.21 & 670 & 71 & 0.21 & 811 & 41 & 0.21 & 810 & 32 \\
\hline $\mathrm{G}_{9}$ & 0.06 & 625 & 336 & 0.09 & 796 & 48 & 1.01 & 928 & 44 & 0.08 & 1025 & 63 \\
\hline $\mathrm{G}_{10}$ & 0.18 & 806 & 43 & 0.04 & 996 & 175 & 0.13 & 1097 & 26 & 0.19 & 1159 & 53 \\
\hline $\mathrm{G}_{11}$ & 0.07 & 1053 & 39 & 0.02 & 1133 & 81 & 0.17 & 1135 & 115 & 0.08 & 1240 & 32 \\
\hline \multirow[t]{2}{*}{$\mathrm{G}_{12}$} & 0.04 & 1180 & 60 & - & - & - & 0.02 & 1173 & 20 & 1.02 & 1329 & 27 \\
\hline & \multicolumn{3}{|c|}{$\delta=0.6 \times 10^{-4} \%$} & \multicolumn{3}{|c|}{$\delta=0.04 \%$} & \multicolumn{3}{|c|}{$\delta=0.3 \%$} & \multicolumn{3}{|c|}{$\delta=0.1 \%$} \\
\hline
\end{tabular}

wavelength $\lambda_{s}$, in expression (22). In the literature [24], a rather weak monotonic dependence of $g_{R}$ on the pumping frequency just at the Stokes wavelength is often referred to as the Raman gain scaling by the pumping wavelength. It is appropriate to be taken separately from the spectroscopic simulation of Raman gain profiles. In fact, the main frequency dependence in a Raman gain profile is given by the imaginary part of the nonlinear polarizability $\chi^{(3)}(18)$ due to the resonant denominator (14), which arises in a phonon harmonic oscillator and leads to the oscillating mode lineform with the form-factor (20) or as the Gaussian function (21).

The physical sense of the Gaussian decomposition procedure for a Raman gain spectrum is substantiated by the amorphism of a core material in all investigated fibers. Due to the amorphous nature of both fused silica and other fibers, each damped oscillator (6) with a resonance of the vibrational coordinate $\mathbf{Q}$ at the frequency $\Omega_{v}$ [according to (11)] has an arbitrary orientation relative to its neighbors, which introduce stochastic perturbations to the frequency of this oscillator. As a result, we should expect the formation of a Gaussian profile for the superposition of a large number of narrow spectral maxima with a nor- mal distribution of random values of the frequencies of these oscillators. Therefore, we have decomposed all Raman gain profiles into several spectral components of the Gaussian type:

$$
g_{R}(\omega)=g_{R \max } \sum_{i=1}^{N_{m}} A_{i} \exp \left[-\left(\omega-\Omega_{v i}\right)^{2} / \Gamma_{i}^{2}\right]=
$$

$=g_{R \max } \varphi(\omega)$

where $\varphi(\omega)$ is an analytic function of the frequency $\omega$ corresponding to a normalized Raman gain profile, $g_{R \max }$ is the maximum value of the Raman gain coefficient; $N_{m}$ is the number of components; $A_{i}$ and $\omega_{v, i}$ are the amplitude and central frequency of the $i$-th Gaussian component, respectively, $\Gamma_{i}=$ $\Delta \omega_{i} /(2 \sqrt{\ln 2}) \approx 0.6 \Delta \omega_{i}$, where $\Delta \omega_{i}$ is the full width at half the maximum for the $i$-th Gaussian profile, which is usually used in spectroscopy.

The main task of the decomposition is to achieve the best fitting of the form-factor $\varphi(\omega)$ to the experimental Raman gain profile of each fiber presented in Fig. $5, a-d$. The problem in practice was solved by finding an optimal set of $3 \times N_{m}$ parameters from (27), by using the computer procedure of nonlinear approximation by the Levenberg-Marquardt method. This 
method uses the algorithm to search for the minimum sum of the least squares by the method of rapid descent to the quadratic minimization of deviations of experimental points from the function $\varphi(\omega)$ defined by $(27)$. With a proper choice of the initial approximation, this method allows us to obtain very good practical results in approximating the Raman amplification spectrum throughout the Stokes shift frequency range from 0 to $1400 \mathrm{~cm}^{-1}$. The numerical values of multicomponent decomposition parameters are presented in the table, and the approximation accuracy of experimental profiles for all $4 \mathrm{SMF}$ types is demonstrated in Fig. 6-9.

The decomposition procedure involving these formation features of rather complex Raman gain profiles in amorphous fibers is represented with two aspects: fundamental and applied. At first, it is the spectroscopic aspect, and the decomposition is considered as a possible method to divide the density of oscillatory states of molecular nanocomplexes into well-defined contributions. In the second applied aspect of decomposition, the goal is to construct the simplest function $g_{R}(\omega)$ corresponding to the experimental Raman gain profile with maximum precision.

The numerical data of the spectroscopic decomposition are presented in the table. They can serve as the interpretation basis of the peculiarities of phonon spectra of the fiber cores forming the Raman gain profiles. For example, all Raman bands in pure $\mathrm{SiO}_{2}$ visible in Fig. 6 with frequencies less than $1053 \mathrm{~cm}^{-1}$ or $1180 \mathrm{~cm}^{-1}$ belong [23], mainly, to fundamental onephonon oscillations. The area of multiphonon overtones and composite tones extends from the line with a frequency of $1180 \mathrm{~cm}^{-1}$ or $1460 \mathrm{~cm}^{-1}$ to a frequency of $2600 \mathrm{~cm}^{-1}$ and above. It remains unclear whether the $1180 \mathrm{~cm}^{-1}$ line corresponds to a one- or two-phonon state.

The main asymmetric maximum of the Raman gain in the pure $\mathrm{SiO}_{2}$ (Fig. 6) at a frequency of $440 \mathrm{~cm}^{-1}$ is formed, according to our data, from at least three of the most intense Gaussian components indicated in the table as $G 3 \rightarrow 359 \mathrm{~cm}^{-1}, G 4 \rightarrow 408 \mathrm{~cm}^{-1}$, and $G 5 \rightarrow 465 \mathrm{~cm}^{-1}$ with damping constants of $182 \mathrm{~cm}^{-1}, 68 \mathrm{~cm}^{-1}$, and $49 \mathrm{~cm}^{-1}$, respectively. The second peak of the Raman gain in pure $\mathrm{SiO}_{2}$ at a frequency of $\sim 495 \mathrm{~cm}^{-1}$ is formed by the rather stable corresponding phonon mode $G 6$ with the damping constant equal to $17 \mathrm{~cm}^{-1}(\sim 50 \mathrm{GHz})$. It should

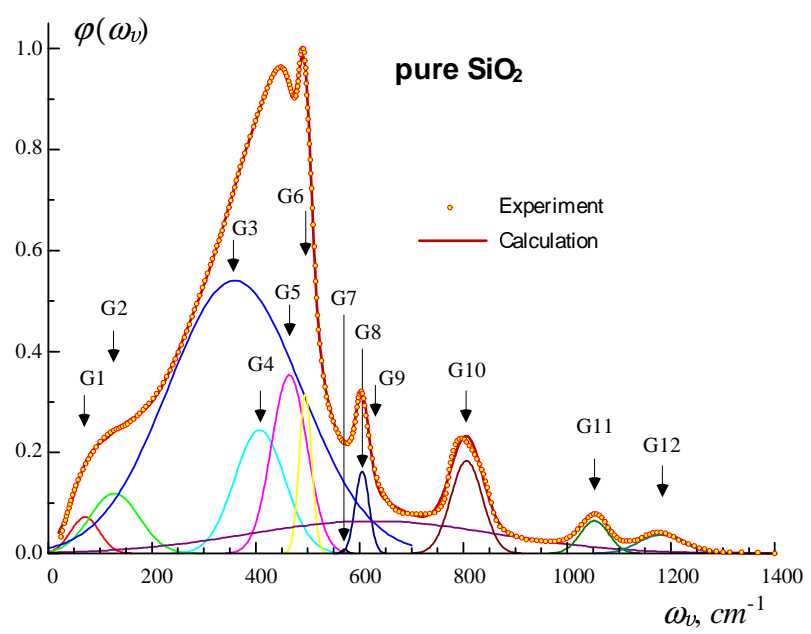

Fig. 6. Decomposition results of a Raman gain profile in a pure silica fiber in the Stokes shift region $0-1400 \mathrm{~cm}^{-1}(0-$ $42 \mathrm{THz})$

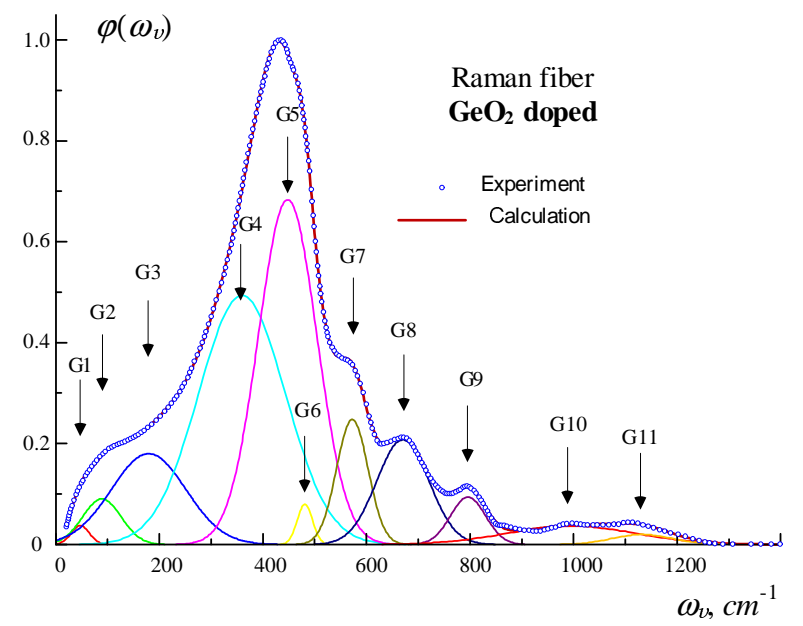

Fig. 7. 11-mode decomposition of Raman gain profiles in a germanosilicate fiber in the Stokes shift region $0-1400 \mathrm{~cm}^{-1}$ (0-42 THz)

be noted that the lifetime of this oscillation almost an order of magnitude longer than the lifetime of its neighboring modes, allows maintaining the stable synchronization conditions (9) required for the coherent amplification of a Stokes wave in the single-pass Raman amplification process. This may be an argument to explain the results of previous experiments [25], where the maximum of a single-pass amplified Stokes wave was observed just at the $495-\mathrm{cm}^{-1}$ frequency of the Stokes shift with the $\mathrm{kW}$-power pulse pumping. 


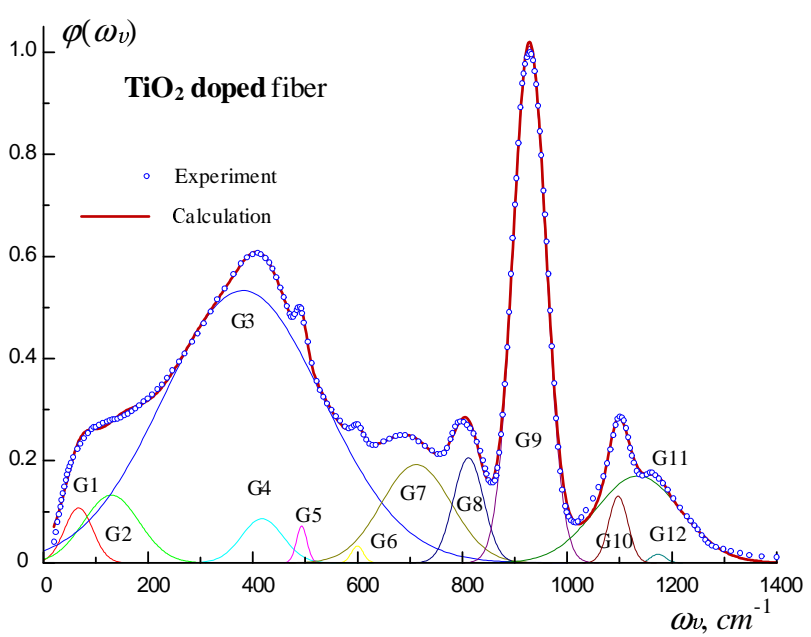

Fig. 8. 12-mode decomposition of Raman gain profiles in a $\mathrm{TiO}_{2}$-doped fiber in the Stokes shift region $0-1400 \mathrm{~cm}^{-1}\left(0_{-}\right.$ $42 \mathrm{THz}$ )

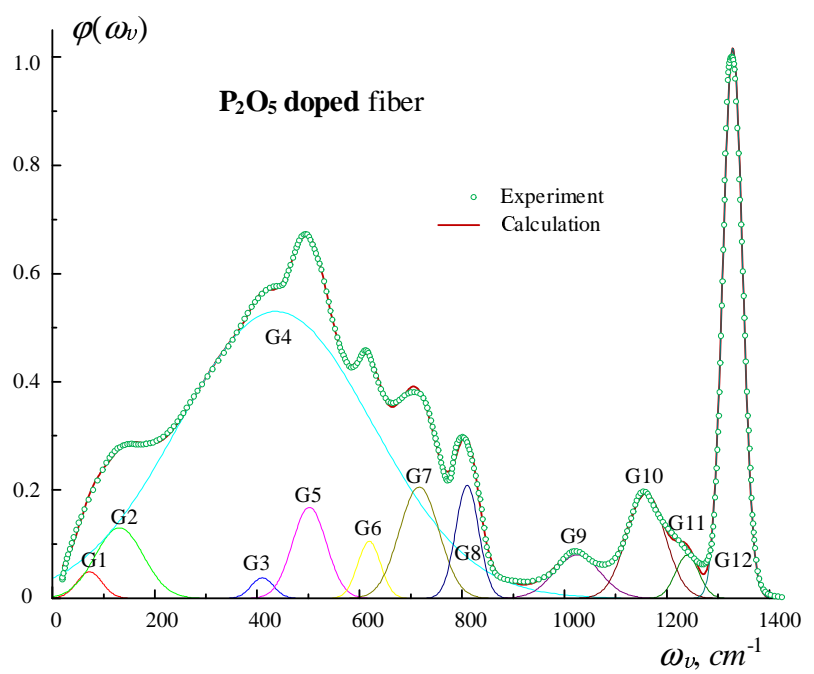

Fig. 9. 12-mode decomposition of Raman gain profiles in a $\mathrm{P}_{2} \mathrm{O}_{5}$-doped fiber in the Stokes shift region $0-1400 \mathrm{~cm}^{-1}(0-$ $42 \mathrm{THz}$ )

The gain profile in a Raman fiber (doped with $\sim 25 \% \mathrm{GeO}_{2}$ ) is notably different from the profile in pure $\mathrm{SiO}_{2}$, as shown in Fig. 7, and 11 Gaussian components were sufficient for its decomposition. The main deformation of a Raman gain profile is due to a sharp increase in the relative intensity of mode $G 5 \rightarrow 448 \mathrm{~cm}^{-1}$, and this is most likely a result of the coupling of two separate modes $G 4$ and $G 5$ observed in the spectrum of pure $\mathrm{SiO}_{2}$. This is confirmed by the average value of the oscillation frequency and the extension of the entire band. The narrow 481$\mathrm{cm}^{-1}$ line becomes more damped, its frequency is lowered by $15 \mathrm{~cm}^{-1}$, the relative intensity is reduced by 4 times, and, therefore, this mode slightly affects the spectral profile. The low-frequency profile slope is formed by two main components $G 3 \rightarrow 179 \mathrm{~cm}^{-1}$ and $G 4 \rightarrow 306 \mathrm{~cm}^{-1}$ with damping constants of $102 \mathrm{~cm}^{-1}$ and $119 \mathrm{~cm}^{-1}$, respectively. The result of such profile rearrangement with $\mathrm{GeO}_{2}$ dopants in the fiber core is one separate rather wide maximum in the 410-450$\mathrm{cm}^{-1}$ region with a simultaneous notable increase in the value of $g_{R \text { max }}$.

The Raman gain profile in a $\mathrm{TiO}_{2}$-doped fiber together with 12 Gaussian components of its decomposition is presented in Fig. 8. The most powerful oscillatory mode $G 9 \rightarrow 928 \mathrm{~cm}^{-1}$ with a damping constant of $44 \mathrm{~cm}^{-1}$ is located in the impurity region of Stokes shifts. The relatively slight damping, as for amorphous materials, testify to the enough large transparency of $\mathrm{TiO}_{2}$-doped glass for the propagation of optical phonons at frequencies $\omega_{v}=930 \mathrm{~cm}^{-1}=$ $=28 \mathrm{THz}$. On the other hand, the full width of this band of the Raman gain is greater than $2 \mathrm{THz}$, and it is quite enough for many applications. In addition, the phonon spectrum of the glass matrix is noticeably rebuilt by the impurities, and its structurization, according to our data, consists in the formation of a powerful oscillatory mode $G 3 \rightarrow 381 \mathrm{~cm}^{-1}$ with a damping constant equal to $215 \mathrm{~cm}^{-1}$. As a result, the Raman gain coefficient is much higher compared with pure $\mathrm{SiO}_{2}$ in the actual Stokes shift area from $100 \mathrm{~cm}^{-1}$ to $600 \mathrm{~cm}^{-1}(3-18 \mathrm{THz})$.

The result of the decomposition of the Raman gain profile in a $\mathrm{P}_{2} \mathrm{O}_{5}$-doped fiber is shown in Fig. 9. The tendency of the $\mathrm{P}_{2} \mathrm{O}_{5}$ impurity to influence the formation of a Raman gain profile in silica glass is very similar to that of $\mathrm{TiO}_{2}$ with certain quantitative differences. The main maximum of the Raman gain coefficient is provided by the oscillating mode $G 12 \rightarrow 1329 \mathrm{~cm}^{-1}$ with the damping constant equal to $27 \mathrm{~cm}^{-1}$. According to our data, the significant contribution to the Raman gain in the domain of the own phonon spectrum of the glass matrix gives mode $G 4 \rightarrow 437 \mathrm{~cm}^{-1}$ with the damping constant equal to $268 \mathrm{~cm}^{-1}$, and this significantly expands the spectral range of applications of $\mathrm{P}_{2} \mathrm{O}_{5}$-doped fibers.

In general, the determination of the damping constant $\Gamma_{i}$ for all Gaussian components allows us to quantitatively estimate the damping degree and the 
lifetime of molecular vibrations, which determine the shape of the Raman spectrum in a doped glass. A common feature of all fibers based on silica glass is the presence of fundamental vibrations with a damping constant $\Gamma_{i} \sim 100 \mathrm{~cm}^{-1}=3 \mathrm{THz}$. Consequently, the attenuation of the principal oscillatory modes forming the Raman gain profiles in the fibers gives the value of the characterized time $\tau_{i} \simeq 1 / \Gamma_{i} \simeq 0.3 \times 10^{-12} \mathrm{~s}$ for the renewal time of the thermodynamic equilibrium or the transients time for Raman amplifiers and lasers.

On the one hand, the femtosecond scale of the relaxation time of the Raman processes provides the possibility for the practical use of FRA and FRL in the terahertz band of operating frequencies. On the other hand, this time interval specifies the limits of the applicability of the quasistationary approximation and, consequently, Eqs. (24) and (25) for the simulation of telecommunication systems with terabit carrying capacity.

However, the presence of a large number of vibrational modes, which strongly overlap, makes it practically impossible to directly separate the Raman bands in the experimental spectrum in all investigated objects. This complicates not so much the computational procedure, as the possibility of an unambiguous interpretation of the obtained results. With the proper choice of the initial approximation for the decomposition with the use of 10-15 Gaussian components, the convergence of the LevenbergMarquard method is provided in real time, and it does not require any extraordinary computational resources. But the optimal set of decomposition parameters obtained as a result of the calculations cannot be guaranteed as the only possible one.

At the same time, although the decomposition uncertainty may cause certain complications in the fundamental interpretation of the vibrational states of molecular complexes, it practically does not affect the applied aspect of the presented calculations. We note that the overwhelming majority of experimental points of the measured spectrum are directly placed on the approximation curves of all Raman gain profiles. Moreover, the deviation of the calculated curves $g_{R \max } \cdot \varphi(\omega)$ exceeding $1 \%$, have only individual unit points of the experimental spectrum at small values of $g_{R}$. From the point of view of the application of the presented model, we can assume that the approximation is practically exact, since the mean square devi- ation is approximately an order of magnitude smaller than the typical accuracies of measurement and spectral processing, as can be seen from the comparison of the data depicted in Figs. 6-9, respectively.

Indeed, the ratio of the integral intensities of the calculated, $I_{G}$, and measured, $I_{0}$, Stokes spectra $\delta=$ $\left(I_{G}-I_{0}\right) / I_{0} \times 100 \%$ given in the table is significantly less than $1 \%$ for all investigated fibers. All integration of functional dependences for data in the table was performed in the range from 0 to $1400 \mathrm{~cm}^{-1}$.

Note that the accuracy of the setting of Raman gain profiles will be of fundamental importance in the simulation of the Raman photonic devices. For example, the telecommunication systems containing 192 channels with wavelength division [26] or even 273 channels [27], which may have the pumping source with more than 10 wavelengths, were described. Consequently, the system of equations (24), (25) becomes a system for several hundred Stokes waves with a dozen pumping waves. In addition, the correlation between the coefficients of amplification and attenuation of the form (26) will determine the singular points, at which the fundamental solution of each differential equation changes fundamentally. Therefore, regardless of the algorithms of solution of such complex systems, any systematic errors in the definition of $g_{R}(\omega)$ cause various kinds of instabilities of calculations, and they become completely unacceptable. In particular, this explains the unsatisfactory results of the simulation of multichannel FRA systems in the case of neglecting the frequency dependence $g_{R}(\omega)$ or its approximation by the very simple functions of the triangle type.

Thus, the spectral Raman gain profile is a key parameter for describing the process of Raman light amplification, and its determination forms the fundamental basis for the design of both high-performance FRAs and FRLs with a band of up to $13 \mathrm{THz}$ in the most common fibers based on silica glass.

\section{Results of Simulation of a Raman Gain Profile and Their Application}

First of all, we note that the Raman gain coefficient in the literature (see [9]) is sometimes given in $\mathrm{dB} /(\mathrm{km} \cdot \mathrm{W})$, and its numerical value differs from the above-introduced term $g_{R}(\omega)$. The value of $g_{0}$ is calculated as

$g_{0}[\mathrm{~dB} /(\mathrm{km} \cdot \mathrm{W})]=\frac{G[\mathrm{~dB}]}{L_{\mathrm{eff}} P}$ 


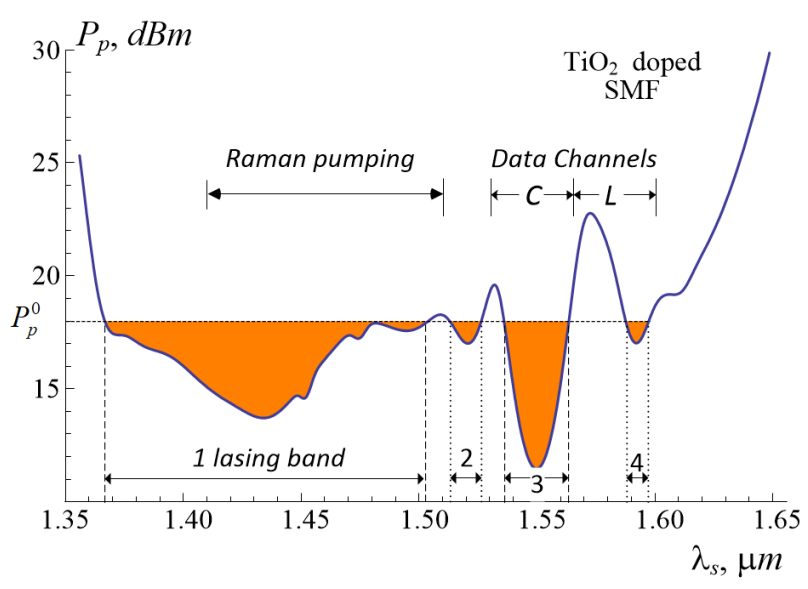

Fig. 10. Four lasing bands are formed in $\mathrm{TiO}_{2}$ doped fiber at $\lambda_{p}=1.35 \mu \mathrm{m}$ and pumping power $P_{p}=18 \mathrm{dBm}$

by the measured value of the so-called on/off gain of $G[\mathrm{~dB}]$ of the weak signal (the ratio of the signal powers for the switched-on and switched-off pumping), $P$ is the pumping power, and the effective fiber length $L_{\mathrm{eff}}=\left[1-\exp \left(-\alpha_{p} L\right)\right] / \alpha_{p}$, where $\alpha_{p}$ is the optical fiber loss at the pumping wavelength. For large $L$, when $\alpha_{p} L \gg 1$, we have $L_{\text {eff }} \simeq 1 / \alpha_{p}$. Note that Eq. (28) is based on the weak signal assumption, and it is actually used an approximate solution of system (24), (25) for a fixed pump, when the pump power depletion is neglected [16]. Furthermore, the measured values of $g_{0}[\mathrm{~dB} /(\mathrm{km} \cdot \mathrm{W})]$ are dependent not only on their own Raman gain, but also on the additional linear parameters concerning the pump power, optical fiber losses at the pumping wavelength, and so on. Therefore, it is often necessary, except for the recalculation from $g_{0}$ into $g_{R}$, to consider corrections to the above restrictions for the accurate use of the $g_{0}[\mathrm{~dB} /(\mathrm{km} \cdot \mathrm{W})]$ term in the simulation of optical waveguide gain processes, in particular, when the system of equations of type $(24),(25)$ is used. It is the invariance of the profile $g_{R}(\omega)$ with respect to the linear fiber parameters and the pumping features making it more suitable for the simulation of the Raman photonic devices.

The most expressive differences between the spontaneous and stimulated Raman spectra in the lowfrequency Stokes shift region discussed in Subsections 1.5 and 2.1 in detail are very useful for experimental studies of FRA noise parameters. In particular, the amplified spontaneous emission has been analyzed for the idle mode (without an external optical signal)
[28], [29], and a mixture of the amplified and spontaneous noises is observed at the output of the investigated fiber. As the pump power increases, the exponential growth in the amplified noise as compared with a non-coherent Stokes noise background independent of the pumping is observed [30]. By the careful analysis of a deformation of the noise spectra under the pumping influence, we were able to separate the coherent amplified Raman noise from the background of the spontaneous Stokes noise, which is discussed in [31], [32] in more details.

On the other hand, the extremely wide frequency band of the single Raman gain profile $(\sim 4 \mathrm{THz}=$ $=130 \mathrm{~cm}^{-1}$ for pure $\mathrm{SiO}_{2}$; Fig. 5, $a$ ), is a basis of the modern technology for the FRA design with a unique wide band of operating frequencies. Using the pumping on several wavelengths, the high-quality FRA of light in the band more than $10 \mathrm{THz}$ with a very low level of own noises was demonstrated $[12,13]$. The completely fiber-based implementation of $10 \mathrm{FRL}$ is presented in [12], where a $6 \lambda \mathrm{FRL}$ provides a uniform amplification of Stokes waves in $\mathrm{C}+\mathrm{L}$ transparency windows. The internal resonator on the fiber Braggs grating in the Raman $\left(\mathrm{GeO}_{2}\right.$-doped) fiber performs a 4 -fold Stokes conversion on $\sim 420 \mathrm{~cm}^{-1}$ of the pumping wavelength from $1100 \mathrm{~nm}$ to $1347 \mathrm{~nm}$. A certain alternative to $\mathrm{GeO}_{2}$-doped fibers, in our opinion, can present a fiber with a $\mathrm{TiO}_{2}$ dopant [33].

Figure 10 shows the modeling results for the full transparency function (26) in a $\mathrm{TiO}_{2}$-doped fiber at $\lambda_{p}=1.35 \mu \mathrm{m}$, which demonstrates the possibility of forming several lasing areas. In particular, at a relatively low pumping power equal to $P_{p}=18 \mathrm{dBm}$, it is possible, as one can see in Fig. 10, to form at least four bands of laser generation across the wavelength range from $1.37 \mu \mathrm{m}$ to almost $1.6 \mu \mathrm{m}$. Note that such wide tuning range of the FRL generation line exceeds almost twice the band of a standard Raman fiber, mainly due to the impurity domain on the Raman gain profile in a $\mathrm{TiO}_{2}$-doped fiber. From the practical point of view, the obvious advantages of $\mathrm{TiO}_{2}$-doped fibers include the possibility to obtain a laser generation both at the FRA pumping wavelengths and the signals carrying information in the transparency windows of the telecommunication fibers.

Some application examples of the doped singlemode fibers as FRA active media are given in [8]. The output power in a fiber Raman laser with a resonator on Bragg gratings with the use of a phosphor sili-

ISSN 2071-0194. Ukr. J. Phys. 2018. Vol. 63, No. 8 
cate fiber with a content of $\mathrm{P}_{2} \mathrm{O}_{5}$ of $13 \%$ molecular weight $(\Delta n=0.011)$ and $200 \mathrm{~m}$ in length was $2.3 \mathrm{~W}$ at $\lambda_{s}=1240 \mathrm{~nm}$ with a pump power of $3.5 \mathrm{~W}$ at $1.06 \mu \mathrm{m}\left(\mathrm{Nd}^{3+}\right)$. Hence, the quantum efficiency was realized to be $77 \%$, by using a Stokes shift frequency of $1330 \mathrm{~cm}^{-1}$. Simultaneously, there are even more serious applied possibilities [34], as indicated in the Fig. 5 for the Raman gain profiles in doped fibers for both multiwave FRL and for ultra wideband Raman amplification of optical signals.

\section{Conclusions}

Spectroscopic features of Raman gain profiles in four types of single-mode fibers based on silica glass are analyzed by their extraction from the experimental spontaneous spectra with successive multimode decomposition, by using Gaussian-type components. The justification of the method is based on the oscillatory model of Raman active vibrations of molecular nanocomplexes in amorphous glass with regard for both quantum and semiclassical approaches. It is shown that the temperature changes of the Raman spectra are concentrated in the low-frequency region of the spectrum. At $T=300 \mathrm{~K}$, these changes are more than an order of magnitude exceed the corresponding components of the Raman gain profile at frequencies $\leq 20 \mathrm{~cm}^{-1}$, but they are became practically invisible at frequencies above $800 \mathrm{~cm}^{-1}$.

It has been found that the nonlinear spectrum of the Raman amplification is much more affected by alloying dopants, by an order of magnitude, as compared with the changes of linear parameters of the fiber core, since the corresponding changes in the refractive index are $\Delta n \simeq 0.01$. The Raman gain profiles are strongly deformed due to the significant restructuring of the vibrational spectra of molecular nanocomplexes of the cores of fibers under the influence of relatively small dopant concentrations. As a result of the Gaussian decomposition with the use of 12 components in pure $\mathrm{SiO}_{2}, \mathrm{P}_{2} \mathrm{O}_{5}$, and $\mathrm{TiO}_{2}$ and using 11 components in a $\mathrm{GeO}_{2}$-doped fiber, it has been established that the main vibrational modes of the glass matrix in fibers, which form the Raman gain profiles, are considerably varied with all oscillation parameters. These changes are several times by the amplitude and about tens of $\mathrm{cm}^{-1}$ by the frequency position and damping constants. Therefore, the average value of the relaxation time of the stimulated Raman processes is $\tau_{i} \simeq 300 \mathrm{fs}$, and this provides, firstly, a possibility of the practical use of Raman amplifiers and light generators in the terahertz band of operating frequencies. Second, such femtosecond time interval indicates the fundamental limit of the quasistationary approximation applicability, namely, for the basic coupled equations describing the nonlinear wave interactions in the Raman effect for the modeling of telecommunication systems with terabit capacity.

The decomposition allows us to obtain the almost exact fitting of experimental data and provides a reliable basis for calculating the main FRA parameters. A simple analytic form of approximating functions in the form of a linear combination of exponentials is an essential advantage of the presented method. It may be useful for the modeling of gain parameters, in particular, for the determination of the spectral density of optical noises and lasing bands for the given pumping parameters, for the optimization of the size of a group delay in the multiwave pumped FRAs, and for the creation of appropriate correction devices.

The work was carried out with partial financial support from the Ministry of Education and Science of Ukraine (Reg. No. 0116U002564); the presented results were obtained during the implementation of the budget theme No. 16BF052-04 at Taras Shevchenko National University of Kyiv.

1. W. Shi, Q. Fang, X. Zhu, R.A. Norwood, N. Peyghambarian. Fiber lasers and their applications. Appl. Opt. 53, 6554 (2014)

2. D.J. Richardson, J. Nilsson, W.A. Clarkson. High-power fiber lasers: current status and future perspectives. J. Opt. Soc. Am. B 27, 63 (2010).

3. M.N. Zervas, C.A. Codemard. High power fiber lasers: A review. IEEE J. Sel. Top. Quantum Electron. 20, 219 (2014).

4. P. Zhou, H. Xiao, J. Leng, J. Xu, Z. Chen, H. Zhang, Z. Liu. High-power fiber lasers based on tandem pumping. J. Opt. Soc. Am. B 34, A29 (2017).

5. P. Ma, H. Zhang, L. Huang, X. Wang, P. Zhou, Z. Liu. Kilowatt-level near-diffraction-limited and linear-polarized ytterbium-Raman hybrid nonlinear amplifier based on polarization selection loss mechanism. Opt. Express 23 (20), 26499 (2015).

6. Q. Xiao, P. Yan, D. Li, J. Sun, X. Wang, Y. Huang, M. Gong. Bidirectional pumped high power Raman fiber laser. Opt. Express 24 (6), 6758 (2016).

7. Y. Feng, L.R. Taylor, D.B. Calia. $150 \mathrm{~W}$ highly-efficient Raman fiber laser. Opt. Express 17 (26), 23678 (2009).

8. E.M. Dianov, A.M. Prokhorov. Medium-power CW Raman fiber lasers. IEEE J. Sel. Top. Quant. Electron. 6, 1022 (2000). 
9. E.M. Dianov. Advances in Raman Fibers. J. Lightwave Techn. 20, 1457 (2002).

10. P.A. Korotkov, G.S. Felinskyi. Fiber SRS lasers with continuous action. Ukr. Fiz. Zh. Oglyady 4 (1), 36 (2007).

11. J. Bromage, K. Rottwitt, M.E. Lines. A method to predict the Raman gain spectra of germanosilicate fibers with arbitrary index profiles. IEEE Photon. Techn. Lett. 14, 24 (2002).

12. M.D. Mermelstein, C. Horn, S. Radic, C. Headley. Six wavelength Raman fiber laser for C- and L-band Raman amplification and dynamic gain flattening. Electron. Lett. 38, 636 (2002).

13. P.A. Korotkov, G.S. Felinskyi. SRS amplification of light in single-mode quartz fibers. Ukr. Fiz. Zh. Oglyady 6 (2), 103 (2009).

14. L. Zhang, C. Liu, H. Jiang, Y. Qi, B. He, J. Zhou, X. Gu, and Y. Feng. Kilowatt ytterbium-Raman fiber laser. Opt. Express 22 (15), 18483 (2014).

15. G.S. Felinskyi, P.A. Korotkov. Raman threshold and optical gain bandwidth in silica fibers. Semicond. Phys. Quant. Electr. Optoelectr. 11, 360 (2008).

16. V.I. Grygoruk, P.A. Korotkov, G.S. Felinskyi. Nonlinear and Laser Processes in Optical Fibers. (Kyiv Univ., 2009) (in Ukrainian).

17. L.D. Landau, E.M. Lifshitz, Mechanics (Butterworth Heinemann, 2001).

18. R.H. Stolen, C. Lee, R.K. Jain. Development of the stimulated Raman spectrum in single-mode fibers. J. Opt. Soc. Am. B 1, 652 (1984).

19. R.H. Stolen, M.A. Bosch. Low frequency and lowtemperature Raman scattering in silica fibers. Phys. Rev. Lett. 48, 805 (1982).

20. P. Voss, Y. Su, P. Kumar, M. Vasilyev. Photon statistics of a single mode of spontaneous Raman scattering in a distributed Raman amplifiers. In Opt. Fiber Commun. Conf. (IEEE, 2001), p. WDD23

21. G.S. Felinskyi, I.V. Serdeha, V.I. Grygoruk. $\mathrm{TiO}_{2}$-doped singlemode fiber as active medium for Raman lasers. In Proc. Int. Symp. on Advanced Material Research, ISAMR'2017, Seoul, Korea, March, 17-19 (2017), p. 109

22. G.S. Felinskyi, I.M. Kudin, I.V. Serdeha. Lasing band and Raman gain threshold in $\mathrm{TiO}_{2}$ doped single-mode fiber. In Proc. 2017 IEEE 37th Int. Conf. on Electronics and Nanotechnology (ELNANO), April 18-20, Kyiv, Ukraine (2017), p. 108

23. G.E. Walrafen, P.N. Krishnan. Model analysis of the Raman spectrum from fused silica optical fibers. Appl. Opt. 21 (3), 359 (1982).

24. K. Rottwitt, J. Bromage, A.J. Stentz et al. Scaling of the Raman gain coefficient: applications to germanosilicate fibers. J. Lightwave Technol. 21 (7), 1652 (2003).

25. K.X. Liu, E. Garmire. Understanding the formation of the SRS Stokes spectrum in fused silica fibers. IEEE J. Quant. Electron. 27 (4), 1022 (1991).

26. F.Di Pasquale, F. Meli, E. Griseri, A. Sguazzotti, C. Tosetti, F. Forghieri. All-Raman transmission of $19225-\mathrm{GHz}$ spaced WDM channels at $10.66 \mathrm{~Gb} / \mathrm{s}$ over $30 \times 22 \mathrm{~dB}$ of
TW-RS fiber. IEEE Photon. Technol. Lett. 15 (2), 314 (2003).

27. K. Fukuchi, T. Kasamatsu, M. Morie et al. 10.92-Tbit/s $(273 \times 40 \mathrm{~Gb} / \mathrm{s})$ Tripleband/ Ultra-dense WDM opticalrepeated transmission experiment. In Opt Fiber Commun. Conf. (IEEE, 2001), p. PD24.

28. G.S. Felinskyi. Noise measurement of the backward pumped distributed fiber Raman amplifier. Photoelectronics 18, 16 (2009).

29. G.S. Felinskyi, M. Dyriv. Signal-to-noise analysis in a counter-pumped fiber Raman amplifier. Optica Applicata 44 (4), 493 (2014).

30. G.S. Felinskyi, M.I. Reznikov, S. Fedorchuk. Amplified and spontaneous stokes noise features in a singlemode silica fiber. In Proc. 2018 IEEE 38th Int. Conf. on Electronics and Nanotechnology (ELNANO), April 24-26, Kyiv, Ukraine (2018), p. 201.

31. G.S. Felinskyi, M. Dyriv. Noise suppression phenomenon in fiber Raman amplifier. Measur. Sci. Rev. 15 (3), 107 (2015).

32. G.S. Felinskyi, M.Y. Dyriv. Noise gain features of fiber Raman amplifier. Adv. OptoElectron. 2016, 1 (2016).

33. G.S. Felinskyi, I.V. Serdeha, V.I. Grygoruk. $\mathrm{TiO}_{2}$-doped single-mode fiber as active material for Raman lasers. Key Engin. Mater. 753, 173 (2017).

34. I.V. Serdeha, G.S. Felinskyi, V.I. Grygoruk. Spectroscopic analysis of Raman lasing features in $\mathrm{P}_{2} \mathrm{O}_{5}$ doped singlemode fiber. In Proc. 2018 IEEE 38th Int. Conf. on Electronics and Nanotechnology (ELNANO), April 24-26, Kyiv, Ukraine (2018), p. 209.

Received 30.11.17

\section{I.B. Сердега, В.І. Григорук, Г.С. Фелінсъкий}

\section{СПЕКТРОСКОПІЧНІ ОСОБЛИВОСТІ} ПРОФІЛІВ ВКР ПІДСИЛЕННЯ В ОДНОМОДОВИХ ВОЛОКНАХ НА ОСНОВІ КВАРЦОВОГО СКЛА

\section{$\mathrm{P}$ е $з$ ю м е}

Надано спектроскопічний аналіз розподілу (профілю) підсилення оптичного випромінювання завдяки ефекту вимушеного комбінаційного розсіяння (ВKР) світла в одномодових волокнах на основі кварцового скла в області стоксового зсуву частот від 0 до $1400 \mathrm{~cm}^{-1}$. Для поширених волокон, а саме для чистого $\mathrm{SiO}_{2}$ та легованих домішками $\mathrm{GeO}_{2}, \mathrm{P}_{2} \mathrm{O}_{5}$ i $\mathrm{TiO}_{2}$, профілі ВKР підсилення визначались за експериментальними спектрами спонтанного розсіяння. Наведені аналітичні вирази профілів ВКР підсилення, які отримані шляхом 11-12 модової гауссової декомпозиції та апроксимують експериментальний профіль з точністю не гірше 0,3\%. Результати декомпозиції проаналізовані як $з$ точки зору фундаментальної коливної динаміки молекулярних нанокомплексів в аморфному склі, так і в прикладному аспекті моделювання пристроїв BКР фотоніки. Представлені приклади застосувань запропонованої методики для аналізу шумових параметрів ВКР підсилювачів та багатосмугової генерації у волоконних ВКР лазерах. 